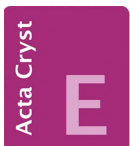

CRYSTALLOGRAPHIC COMMUNICATIONS

ISSN 2056-9890

Received 29 September 2021

Accepted 9 November 2021

Edited by J. T. Mague, Tulane University, USA

Keywords: crystal structure; fluorenonophane; hydrogen bonds.

CCDC reference: 2121128

Supporting information: this article has supporting information at journals.iucr.org/e

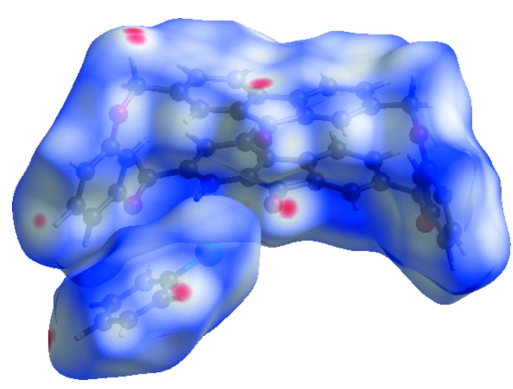

OPEN $\partial$ ACCESS

\section{Fluorenonophane chlorobenzene solvate: molecular and crystal structures}

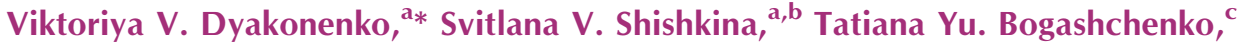 \\ Alexander Yu. Lyapunov ${ }^{\mathrm{c}, \mathrm{d}, \mathrm{e}}$ and Tatiana I. Kirichenko ${ }^{\mathrm{c}}$ \\ aSSI 'Institute for Single Crystals', National Academy of Sciences of Ukraine, 60 Nauky Ave., Kharkiv 61001, Ukraine, \\ ${ }^{b}$ V. N. Karazin Kharkiv National University, 4 Svobody sq., Kharkiv 61112, Ukraine, ' ${ }^{\mathrm{C}}$. V. Bogatsky Physico-Chemical \\ Institute, National Academy of Sciences of Ukraine, 86 Lustdorfskaya doroga, Odesa, Ukraine, 'Enamine Ltd., \\ Chervonotkatska Street 78, Kyiv, 02094 , Ukraine, and ${ }^{\mathbf{e} T a r a s ~ S h e v c h e n k o ~ N a t i o n a l ~ U n i v e r s i t y ~ o f ~ K y i v, ~ V o l o d y m y r s k a ~}$ \\ Street 60, Kyiv, 01601, Ukraine. *Correspondence e-mail: vika@xray.isc.kharkov.com
}

The title compound, $1{ }^{9} H, 7^{9} H-3,5,9,11$-tetraoxa-1,7(2,7)-difluorena-4,10(1,3)dibenzenacyclododecaphane- $1^{9}, 7^{9}$-dione (fluorenonophane), exists as a solvate with chlorobenzene, $\mathrm{C}_{42} \mathrm{H}_{28} \mathrm{O}_{6} \cdot \mathrm{C}_{6} \mathrm{H}_{5} \mathrm{Cl}$. The fluorenonophane contains two fluorenone fragments linked by two $m$-substituted benzene fragments. Some decrease in its macrocyclic cavity leads to a stacking interaction between the tricyclic fluorenone fragments. In the crystal, the fluorenonophane and chlorobenzene molecules are linked by weak $\mathrm{C}-\mathrm{H} \cdots \pi($ ring) interactions and $\mathrm{C}-\mathrm{H} \cdots \mathrm{Cl}$ hydrogen bonds. The $\mathrm{Cl}$ atom of chlorobenzene does not form a halogen bond. A Hirshfeld surface analysis and two-dimensional fingerprint plots were used to analyse the intermolecular contacts found in the crystal structure.

\section{Chemical context}

Discovered at the end of the last century, the ability of cyclophanes to form inclusion complexes makes them the central class of synthetic receptors in molecular recognition processes (Diederich, 1991). Particular attention has been paid to the possibility of cationic cyclophanes with box geometries being involved in strong donor-acceptor interactions leading to the formation of 'guest-host' complexes with different guests (Dale et al., 2016; Barnes et al., 2013; Gong et al., 2010). Previously we have obtained fluorenonophane 1 with two fluorenone fragments linked by rigid xylyl groups (Lukyanenko et al., 2003; Simonov et al., 2006). $\mathrm{X}$-ray diffraction analysis of this cyclophane revealed the box geometry with an open intramolecular cavity and the formation of inclusion complexes with DMF and nitrobenzene (Simonov et al., 2006). The other fluorenonophane obtained by our group, 2, differs from the previous one in the position of the methylene groups, which are located directly at the benzene fragment in $\mathbf{1}$ or fluorenone in $\mathbf{2}$. Fluorenonophane $\mathbf{2}$ forms inclusion complexes with chloroform and bromoform with a $1: 2$ stoichiometry. Moreover, $\mathrm{C}-\mathrm{Cl} \cdots \pi$ and $\mathrm{C}-\mathrm{Br} \cdots \pi$ halogen bonds (Shishkina et al., 2021) are present in the complexes. In contrast to cationic cyclophanes, there are no charged fragments in fluorenonophanes. Continuing our research in this area, we have obtained fluorenonophane $\mathbf{3}$ with a different position of attachment of the benzene rings compared to $\mathbf{2}$ ( $m$ - and $p$-isomers, respectively) and studied its complexation with chlorobenzene. 


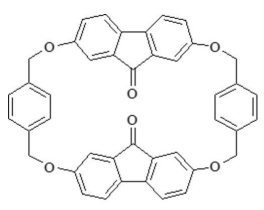

1

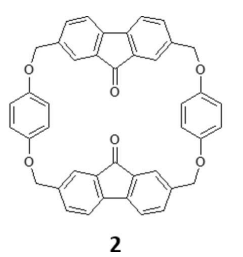

2

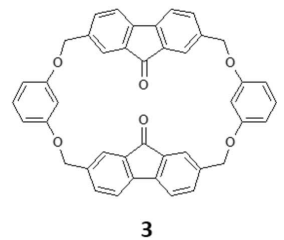

\section{Structural commentary}

Fluorenonophane $\mathbf{3}$ was crystallized from chlorobenzene and exists in the crystal as a solvate in a 1:1 ratio rather than as an inclusion complex. Fluorenonophane $\mathbf{3}$ contains two fluorenone fragments linked by two $m$-substituted benzene fragments (Fig. 1). The macrocycle $\mathbf{3}$ has a boat conformation similar to structure $\mathbf{1}$ [the torsion angles $\mathrm{C} 41-\mathrm{O} 6-\mathrm{C} 1-\mathrm{C} 2$, $\mathrm{C} 37-\mathrm{O} 5-\mathrm{C} 36-\mathrm{C} 33, \mathrm{C} 20-\mathrm{O} 3-\mathrm{C} 22-\mathrm{C} 23$, and $\mathrm{C} 16-\mathrm{O} 2-$ $\mathrm{C} 15-\mathrm{C} 13$ are $-90.6(4), 78.4(4),-80.0$ (4) and $91.6(4)^{\circ}$, respectively]. In structure $\mathbf{3}$, the fluorenone fragments are oriented in the same directions (cis-orientation) while the orientation of these fragments is trans in structures $\mathbf{1}$ and $\mathbf{2}$. meta-Substitution of the two benzene fragments results in a smaller macrocycle cavity as compared to fluorenonophanes $\mathbf{1}$ and 2 with para-substituted benzene fragments. As a result, the two fluorenones are slightly bowed inwards [the dihedral angle between $\mathrm{C} 2-\mathrm{C} 7$ and $\mathrm{C} 8-\mathrm{C} 14$ benzene rings is $12.51(18)^{\circ}$ in one fluorenone while the dihedral angle between the C31$\mathrm{C} 35$ and $\mathrm{C} 23-\mathrm{C} 28$ benzene rings is $9.64(18)^{\circ}$ in the other fluorenone). This can be explained by a $\pi$-stacking interaction between the $\mathrm{C} 10=\mathrm{O} 1$ carbonyl group and the $\mathrm{C} 25 / \mathrm{C} 26 / \mathrm{C} 31 /$ $\mathrm{C} 30 / \mathrm{C} 29$ fluorenone ring [centroid $\mathrm{Cg} 2$, with $\mathrm{O} 1 \cdots \mathrm{Cg} 2=$ 3.469 (3) $\AA, C 10 \cdots C g 2=3.492$ (4) $\AA, C 10=\mathrm{O} 1 \cdots C g 2=$ $\left.81.1(2)^{\circ}\right]$. In contrast to structures $\mathbf{1}$ and $\mathbf{2}$, the macrocycle in
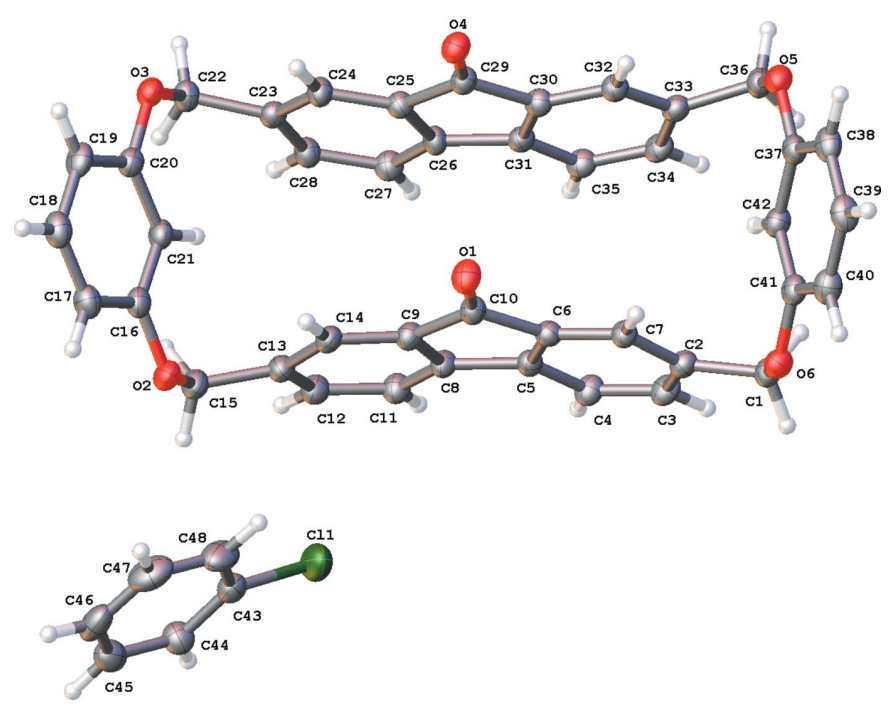

Figure 1

The molecular structure of the title compound showing the atomlabelling scheme. Displacement ellipsoids are drawn at the $50 \%$ probability level.
Table 1

Hydrogen-bond geometry $\left(\AA,^{\circ}\right)$.

$C g 1, C g 2$ and $C g 15$ are the centroids of the $\mathrm{C} 5 / \mathrm{C} 6 / \mathrm{C} 10 / \mathrm{C} 9 / \mathrm{C} 8, \mathrm{C} 25 / \mathrm{C} 29 / \mathrm{C} 30 /$ $\mathrm{C} 31 / \mathrm{C} 26$ and $\mathrm{C} 43-\mathrm{C} 48$ rings, respectively.

\begin{tabular}{lllll}
\hline$D-\mathrm{H} \cdots A$ & $D-\mathrm{H}$ & $\mathrm{H} \cdots A$ & $D \cdots A$ & $D-\mathrm{H} \cdots A$ \\
\hline $\mathrm{C} 18-\mathrm{H} 18 \cdots \mathrm{Cl} 1^{\mathrm{i}}$ & 0.95 & 2.83 & $3.547(4)$ & 133 \\
$\mathrm{C} 35-\mathrm{H} 35 \cdots \mathrm{O} 1^{\mathrm{ii}}$ & 0.95 & 2.58 & $3.491(5)$ & 161 \\
$\mathrm{C} 46-\mathrm{H} 46 \cdots 6^{\mathrm{iii}}$ & 0.95 & 2.55 & $3.418(5)$ & 152 \\
$\mathrm{C} 1-\mathrm{H} 1 A \cdots C g 2^{\text {iv }}$ & 0.99 & 2.95 & $3.610(4)$ & 125 \\
$\mathrm{C} 22-\mathrm{H} 22 A \cdots C g 1^{\mathrm{v}}$ & 0.99 & 2.73 & $3.711(4)$ & 170 \\
$\mathrm{C} 36-\mathrm{H} 36 B \cdots C g 15^{\text {vi }}$ & 0.99 & 2.84 & $3.713(4)$ & 148 \\
\hline
\end{tabular}

Symmetry codes: (i) $x+1, y+1, z$; (ii) $x-1, y, z$; (iii) $x+1, y+1, z+1$; (iv) $x, y-1, z$; (v) $x, y+1, z$; (vi) $x-1, y, z-1$.

structure $\mathbf{3}$ does not contain any molecules inside its cavity. Therefore, the structure under study is a chlorobenzene solvate of fluorenonophane.

\section{Supramolecular features}

In the crystal, the fluorenonophane and chlorobenzene molecules are linked to each other by weak C46-H46...O6 and $\mathrm{C} 18-\mathrm{H} 18 \cdots \mathrm{Cl} 1$ hydrogen bonds while the fluorenophanes are linked by weak C35-H35 . . O1 hydrogen bonds (Table 1), forming stepped ribbons. The ribbons are connected by $\mathrm{C} 1-$ $\mathrm{H} 1 A \cdots C g 2$ and $\mathrm{C} 22-\mathrm{H} 22 A \cdots C g 1$ interactions (Table 1) to give the final three-dimensional structure. The halogen atom does not form a halogen bond in the structure of $\mathbf{3}$, in contrast to the supramolecular complexes studied earlier (Shishkina et al., 2021). The electrostatic potential for chlorobenzene was calculated using the B3LYP/6-311 G(d,p) method. An area with a positive charge ( $\sigma$-hole) was not found in the electrostatic potential map around the halogen atom (Fig. 2). The highest electrostatic potential at the chlorine atom is $-0.08 \mathrm{eV}$. This fact can explain the absence of halogen bonds in the structure of $\mathbf{3}$.

\section{Hirshfeld surface analysis}

Crystal Explorer 17.5 (Turner et al., 2017) was used to analyze interactions in the crystal. Molecular Hirshfeld surfaces mapped over $d_{\text {norm }}$ with a standard (high) surface resolution

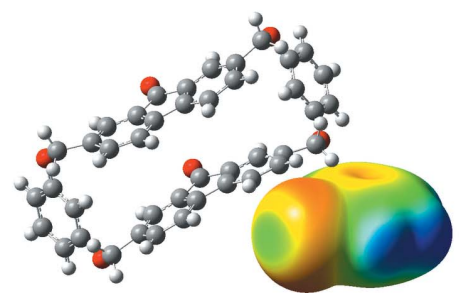

Figure 2

Electrostatic potential map of the chlorobenzene molecule in $\mathbf{3}$ calculated by the B3LYP/6-311 G(d,p) method. 


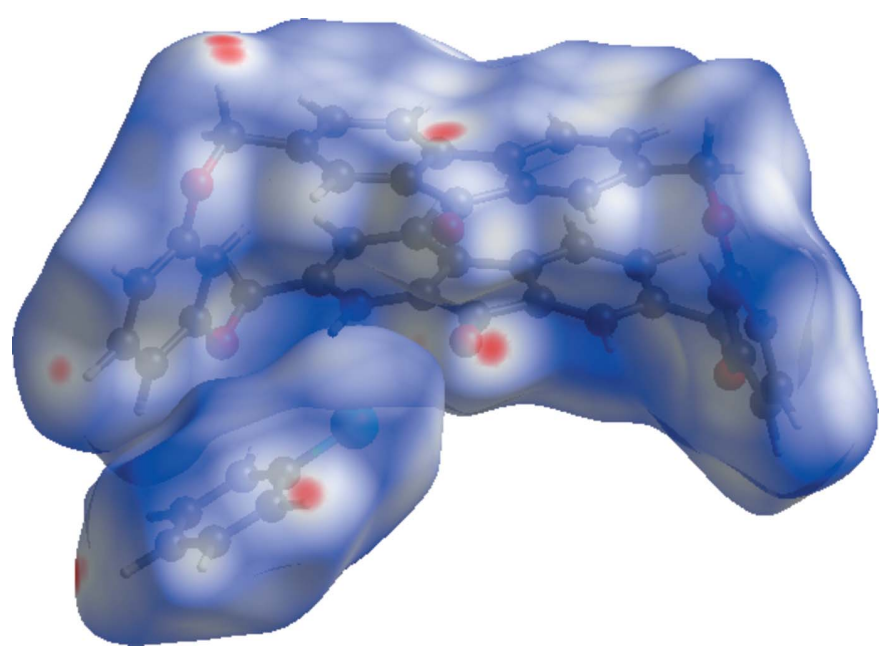

Figure 3

Hirshfeld surface mapped over $d_{\text {norm }}$ showing the conformation of the fluorenonophane and chlorobenzene molecules.

and a fixed colour scale of -0.134 (red) to 1.206 (blue) were generated separately (Fig. 3) for the fluorenonophane and chlorobenzene molecules. The areas in red correspond to contacts that are shorter than the sum of the van der Waals radii of the closest atoms. Thus, the red spots at some hydrogen atoms and at the carbonyl oxygen atom as well as in the area of the five-membered ring indicate the existence of short $\mathrm{C}-\mathrm{H} \cdots \mathrm{O}$ and $\mathrm{C}-\mathrm{H} \cdots \pi$ (ring) contacts.

To evaluate the contribution of the short contacts of different types to the total Hirshfeld surface, two-dimensional fingerprint plots for the fluorenonophane and chlorobenzene molecules were generated (Fig. 4). The contribution from the
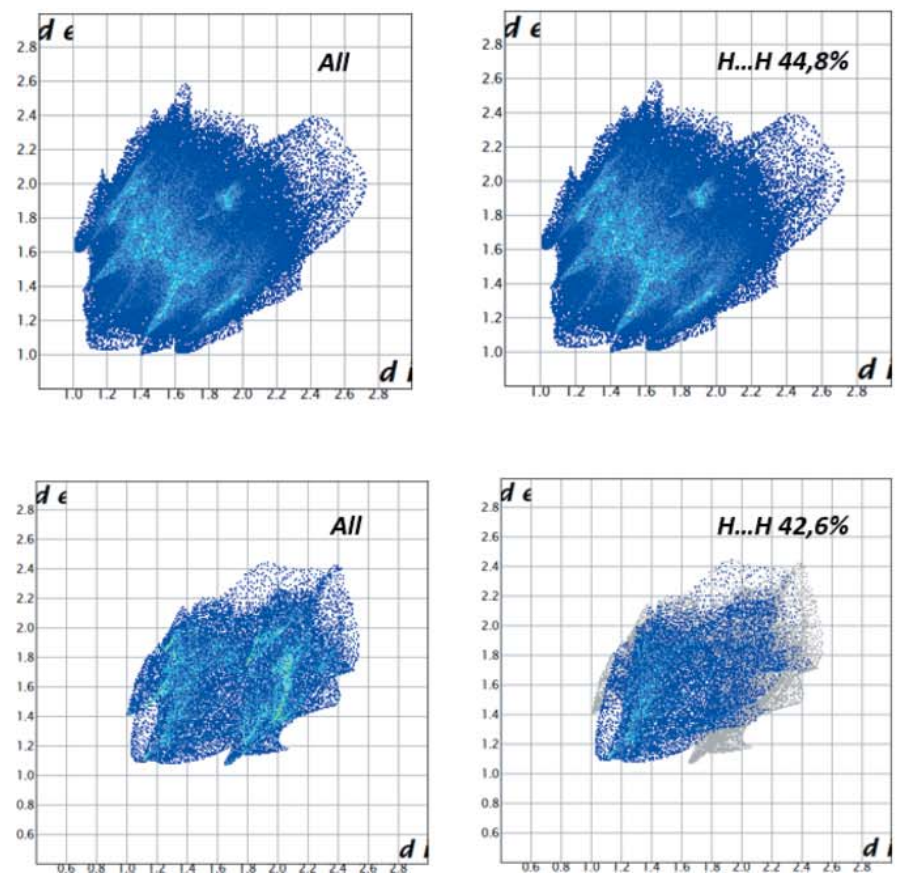
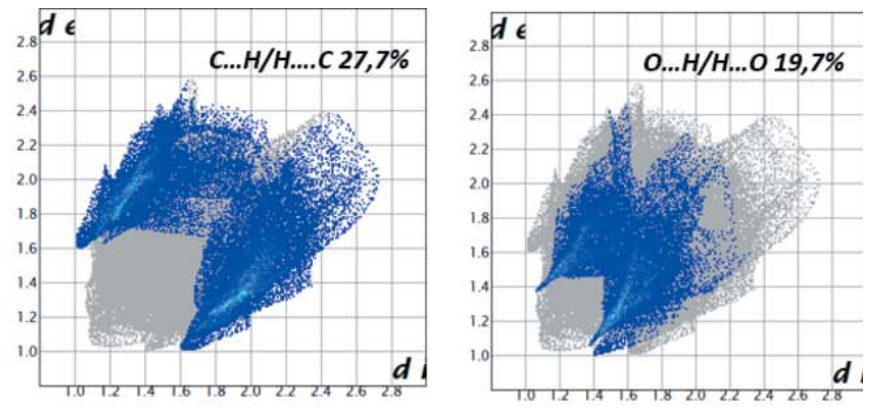

C. $\cdot \mathrm{H} / \mathrm{H} \cdots \mathrm{C}$ contacts corresponding to the $\mathrm{C}-\mathrm{H} \cdots \pi$ (ring) interactions are represented by a pair of sharp spikes $(27.7 \%$ and $25.9 \%$ for fluorenonophane and chlorobenzene, respectively). Analysis of the fingerprint plots also showed a significant contribution from $\mathrm{O} \cdots \mathrm{H} / \mathrm{H} \cdots \mathrm{O}$ contacts $(19.7 \%)$ associated with the $\mathrm{C}-\mathrm{H} \cdots \mathrm{O}$ hydrogen bonds.

\section{Database survey}

A search of the Cambridge Structural Database (CSD, Version 5.42, update of November 2020; Groom et al., 2016) for cyclophanes containing fluorenone and benzene fragments yielded two hits: two structures with fluorenone fragments linked by rigid xylyl groups (CCDC 263272 and CCDC 263273; Simonov et al., 2006). Recently, two more structures with fluorenonophanes linked by para-substituted benzene fragments were published (CCDC 647971 and CCDC 2098245; Shishkina et al., 2021). The structures found are characterized by a larger macrocyclic cavity compared to that in fluorenonophane $\mathbf{3}$.

\section{Synthesis and crystallization}

A solution of $1.75 \mathrm{~g}$ (4.78 mmol) of 2,7-bis(bromomethyl)-9Hfluoren-9-one (Haenel et al., 1985) in $200 \mathrm{~mL}$ of anhydrous DMF was added to a mixture of $0.526 \mathrm{~g}(4.78 \mathrm{mmol})$ of resorcinol and $3.96 \mathrm{~g}(28.7 \mathrm{mmol})$ of $\mathrm{K}_{2} \mathrm{CO}_{3}$ in $270 \mathrm{~mL}$ of anhydrous DMF with stirring under nitrogen for $10 \mathrm{~h}$ at 353$358 \mathrm{~K}$. The reaction mixture was stirred at the same temperature for a further $35 \mathrm{~h}$, cooled and filtered (Fig. 5). The precipitate was washed with DMF and the filtrate was evaporated under reduced pressure. The residue was dissolved
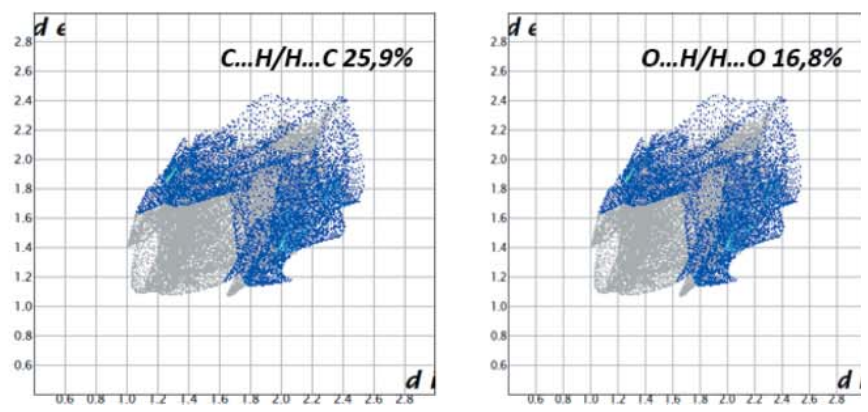

Figure 4

The two-dimensional fingerprint plots for fluorenonophane $\mathbf{3}$ (top) and chlorobenzene (bottom). 


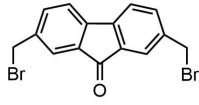

Figure 5

The synthesis of fluorenonophane $\mathbf{3}$

in $\mathrm{CHCl}_{3}$ and washed with an aqueous sodium carbonate solution $(50 \mathrm{~mL})$, then with water $(3 \times 50 \mathrm{~mL})$ to a neutral pH. After drying over $\mathrm{MgSO}_{4}$, the $\mathrm{CHCl}_{3}$ was evaporated under reduced pressure. The product was purified by chromatography on silica gel (Acros $0.060 \div 1 / 5$ ), eluent $\mathrm{CHCl}_{3}-$ EtOH, 500:1. The yield of cyclophane 3 was $0.11 \mathrm{~g}$ (7.2\%), m.p. $>573 \mathrm{~K}$, dec. ${ }^{1} \mathrm{H}$ NMR (DMSO-d $\left.d_{6}\right)$, $\delta$, p.p.m.: $5.25 s\left(\mathrm{CH}_{2}, 8 \mathrm{H}\right)$, 6.46-6.56 $m\left(\mathrm{H}_{2}, \mathrm{H}_{4}, 6 \mathrm{H}\right), 7.04 t\left(\mathrm{H}_{5}, 2 \mathrm{H}, J=8.1 \mathrm{~Hz}\right), 7.18 s$ $(\mathrm{H} a, 4 \mathrm{H}), 7.57 m(\mathrm{H} b, \mathrm{HH}, 8 \mathrm{H})$. MS: FAB, $m / z, 628\left[M+\mathrm{H}^{+}\right]$. Analysis calculated for $\mathrm{C}_{42} \mathrm{H}_{28} \mathrm{O}_{6}: \mathrm{C}, 80.24 ; \mathrm{H}, 4.49$. Found: $\mathrm{C}$, $80.44 ; \mathrm{H}, 4.76 \%$. Crystals were obtained by crystallization of fluorenonophane $\mathbf{3}$ from chlorobenzene.

\section{Refinement}

Crystal data, data collection, and structure refinement details are summarized in Table 2. Carbon-bound $\mathrm{H}$ atoms were added in calculated positions with $\mathrm{C}-\mathrm{H}$ bond lengths of $0.95 \AA$ for $\mathrm{C}-\mathrm{H}, 0.92 \AA$ for $\mathrm{CH}_{2}$ and refined as riding atoms with $U_{\text {iso }}(\mathrm{H})=1.2 U_{\text {eq }}(\mathrm{C})$.

\section{Funding information}

The authors thank the National Academy of Sciences of Ukraine for financial support in the framework of the projects 'New supramolecular systems based on cyclophanes with fluorenone and benzimidazole fragments. Design, synthesis, structure, perspectives' (0120U100122) and 'Functional materials for biomedical purposes based on halogencontaining organic compounds' (0120U102660).

\section{References}

Barnes, J. C., Juríček, M., Strutt, N. L., Frasconi, M., Sampath, S., Giesener, M. A., McGrier, P. L., Bruns, C. J., Stern, C. L., Sarjeant, A. A. \& Stoddart, J. F. (2013). J. Am. Chem. Soc. 135, 183-192.

Dale, E. J., Vermeulen, N. A., Juríček, M., Barnes, J. C., Young, R. M., Wasielewski, M. R. \& Stoddart, J. F. (2016). Acc. Chem. Res. 49, 262-273.

Diederich, F. (1991). Cyclophanes. Cambridge: The Royal Society of Chemistry.

Dolomanov, O. V., Bourhis, L. J., Gildea, R. J., Howard, J. A. K. \& Puschmann, H. (2009). J. Appl. Cryst. 42, 339-341.

Gong, H.-Y., Rambo, B. M., Karnas, E., Lynch, V. M. \& Sessler, J. L. (2010). Nat. Chem. 2, 406-409.
Table 2

Experimental details.

\begin{tabular}{|c|c|}
\hline \multicolumn{2}{|l|}{ Crystal data } \\
\hline Chemical formula & $\mathrm{C}_{42} \mathrm{H}_{28} \mathrm{O}_{6} \cdot \mathrm{C}_{6} \mathrm{H}_{5} \mathrm{Cl}$ \\
\hline$M_{\mathrm{r}}$ & 741.19 \\
\hline Crystal system, space group & Triclinic, $P 1$ \\
\hline Temperature (K) & 100 \\
\hline$a, b, c(\AA)$ & $6.2278(6), 9.6965(8), 14.9822(13)$ \\
\hline$\alpha, \beta, \gamma\left(^{\circ}\right)$ & $105.288(8), 97.126(7), 96.919(7)$ \\
\hline$V\left(\AA^{3}\right)$ & $854.83(13)$ \\
\hline$Z$ & 1 \\
\hline Radiation type & Мо $K \alpha$ \\
\hline$\mu\left(\mathrm{mm}^{-1}\right)$ & 0.17 \\
\hline Crystal size $(\mathrm{mm})$ & $0.6 \times 0.4 \times 0.2$ \\
\hline \multicolumn{2}{|l|}{ Data collection } \\
\hline Diffractometer & Xcalibur, Sapphire3 \\
\hline Absorption correction & $\begin{array}{l}\text { Multi-scan (CrysAlis PRO; Rigaku } \\
\text { OD, 2018) }\end{array}$ \\
\hline$T_{\min }, T_{\max }$ & $0.846,1.000$ \\
\hline $\begin{array}{l}\text { No. of measured, independent and } \\
\text { observed }[I>2 \sigma(I)] \text { reflections }\end{array}$ & $8226,7191,5307$ \\
\hline$R_{\text {int }}$ & 0.028 \\
\hline$(\sin \theta / \lambda)_{\max }\left(\AA^{-1}\right)$ & 0.808 \\
\hline \multicolumn{2}{|l|}{ Refinement } \\
\hline$R\left[F^{2}>2 \sigma\left(F^{2}\right)\right], w R\left(F^{2}\right), S$ & $0.064,0.171,1.03$ \\
\hline No. of reflections & 7191 \\
\hline No. of parameters & 496 \\
\hline No. of restraints & 3 \\
\hline $\mathrm{H}$-atom treatment & $\mathrm{H}$-atom parameters constrained \\
\hline$\Delta \rho_{\max }, \Delta \rho_{\min }\left(\mathrm{e} \AA^{-3}\right)$ & $0.79,-0.42$ \\
\hline Absolute structure & $\begin{array}{l}\text { Flack } x \text { determined using } 564 \\
\quad \text { quotients }\left[\left(I^{+}\right)-\left(I^{-}\right)\right] /\left[\left(I^{+}\right)+\left(I^{-}\right)\right] \\
\quad \text { (Parsons } \text { et al., } 2013)\end{array}$ \\
\hline Absolute structure parameter & $0.19(9)$ \\
\hline
\end{tabular}

Computer programs: CrysAlis PRO (Rigaku OD, 2018), SHELXT (Sheldrick, 2015a), SHELXL2018/3 (Sheldrick, 2015b) and OLEX2 (Dolomanov et al., 2009).

Groom, C. R., Bruno, I. J., Lightfoot, M. P. \& Ward, S. C. (2016). Acta Cryst. B72, 171-179.

Haenel, M. W., Irngartinger, H. \& Krieger, C. (1985). Chem. Ber. 118, 144-162.

Lukyanenko, N. G., Kirichenko, T. I., Lyapunov, A. Yu., Bogaschenko, T. Yu., Pastushok, V. N., Simonov, Yu. A., Fonari, M. S. \& Botoshansky, M. M. (2003). Tetrahedron Lett. 44, 73737376.

Parsons, S., Flack, H. D. \& Wagner, T. (2013). Acta Cryst. B69, 249259.

Rigaku OD (2018). CrysAlis PRO. Rigaku Oxford Diffraction, Yarnton, England.

Sheldrick, G. M. (2015a). Acta Cryst. A71, 3-8.

Sheldrick, G. M. (2015b). Acta Cryst. C71, 3-8.

Shishkina, S. V., Dyakonenko, V. V., Shishkin, O. V., Semynozhenko, V. P., Bogashchenko, T. Yu., Lyapunov, A. Yu. \& Kirichenko, T. I. (2021). Struct. Chem. http://doi.org/10.21203/rs.3.rs-747526/v1

Simonov, Yu. A., Bogashchenko, N. Yu., Pastushok, V. N., Botoshanskii, M. M., Fonar', M. S., Lyapunov, A. Yu. \& Luk'yanenko, N. G. (2006). Russ. J. Org. Chem. 42, 1075-1082.

Turner, M. J., McKinnon, J. J., Wolff, S. K., Grimwood, D. J., Spackman, P. R., Jayatilaka, D. \& Spackman, M. A. (2017). CrystalExplorer17. University of Western Australia. http://Hirshfeldsurface.net 


\section{supporting information}

Acta Cryst. (2021). E77, 1285-1288 [https://doi.org/10.1107/S2056989021011865]

\section{Fluorenonophane chlorobenzene solvate: molecular and crystal structures}

Viktoriya V. Dyakonenko, Svitlana V. Shishkina, Tatiana Yu. Bogashchenko, Alexander Yu. Lyapunov and Tatiana I. Kirichenko

\section{Computing details}

Data collection: CrysAlis PRO (Rigaku OD, 2018); cell refinement: CrysAlis PRO (Rigaku OD, 2018); data reduction: CrysAlis PRO (Rigaku OD, 2018); program(s) used to solve structure: SHELXT (Sheldrick, 2015a); program(s) used to refine structure: SHELXL2018/3 (Sheldrick, 2015b); molecular graphics: OLEX2 (Dolomanov et al., 2009); software used to prepare material for publication: OLEX2 (Dolomanov et al., 2009).

$1^{9} H, 7^{9} H-3,5,9,11-T e t r a o x a-1,7(2,7)$-difluorena-4,10(1,3)-dibenzenacyclododecaphane- $1^{9}, 7^{9}$-dione

chlorobenzene monosolvate

Crystal data

$\mathrm{C}_{42} \mathrm{H}_{28} \mathrm{O}_{6} \cdot \mathrm{C}_{6} \mathrm{H}_{5} \mathrm{Cl}$

$M_{r}=741.19$

Triclinic, $P 1$

$a=6.2278(6) \AA$

$b=9.6965(8) \AA$

$c=14.9822(13) \AA$

$\alpha=105.288(8)^{\circ}$

$\beta=97.126(7)^{\circ}$

$\gamma=96.919(7)^{\circ}$

$V=854.83(13) \AA^{3}$

Data collection

Xcalibur, Sapphire3 diffractometer

Radiation source: fine-focus sealed X-ray tube, Enhance (Mo) X-ray Source

Graphite monochromator

Detector resolution: 16.1827 pixels $\mathrm{mm}^{-1}$

$\omega$ scans

Absorption correction: multi-scan

(CrysAlisPro; Rigaku OD, 2018)

\section{Refinement}

Refinement on $F^{2}$

Least-squares matrix: full

$R\left[F^{2}>2 \sigma\left(F^{2}\right)\right]=0.064$

$w R\left(F^{2}\right)=0.171$

$S=1.03$

7191 reflections

496 parameters
$Z=1$

$F(000)=386$

$D_{\mathrm{x}}=1.440 \mathrm{Mg} \mathrm{m}^{-3}$

Mo $K \alpha$ radiation, $\lambda=0.71073 \AA$

Cell parameters from 1987 reflections

$\theta=3.9-33.0^{\circ}$

$\mu=0.17 \mathrm{~mm}^{-1}$

$T=100 \mathrm{~K}$

Block, colourless

$0.6 \times 0.4 \times 0.2 \mathrm{~mm}$

$T_{\min }=0.846, T_{\max }=1.000$

8226 measured reflections

7191 independent reflections

5307 reflections with $I>2 \sigma(I)$

$R_{\text {int }}=0.028$

$\theta_{\max }=35.0^{\circ}, \theta_{\min }=3.0^{\circ}$

$h=-9 \rightarrow 8$

$k=-7 \rightarrow 15$

$l=-24 \rightarrow 20$

3 restraints

Hydrogen site location: inferred from neighbouring sites

$\mathrm{H}$-atom parameters constrained

$w=1 /\left[\sigma^{2}\left(F_{\mathrm{o}}^{2}\right)+(0.0825 P)^{2}+0.017 P\right]$ where $P=\left(F_{\mathrm{o}}^{2}+2 F_{\mathrm{c}}^{2}\right) / 3$

$(\Delta / \sigma)_{\max }<0.001$ 


$$
\Delta \rho_{\max }=0.79 \text { e } \AA^{-3}
$$$$
\Delta \rho_{\min }=-0.42 \text { e } \AA^{-3}
$$

Absolute structure: Flack $x$ determined using 564 quotients $\left[\left(I^{+}\right)-(I)\right] /\left[\left(I^{+}\right)+\left(I^{-}\right)\right]$(Parsons et al.,2013)

Absolute structure parameter: 0.19 (9)

\section{Special details}

Geometry. All esds (except the esd in the dihedral angle between two 1.s. planes) are estimated using the full covariance matrix. The cell esds are taken into account individually in the estimation of esds in distances, angles and torsion angles; correlations between esds in cell parameters are only used when they are defined by crystal symmetry. An approximate (isotropic) treatment of cell esds is used for estimating esds involving l.s. planes.

Fractional atomic coordinates and isotropic or equivalent isotropic displacement parameters $\left(\AA^{2}\right)$

\begin{tabular}{|c|c|c|c|c|}
\hline & $x$ & $y$ & $z$ & $U_{\text {iso }} * / U_{\text {eq }}$ \\
\hline $\mathrm{O} 1$ & $0.3386(4)$ & $0.2409(3)$ & $0.30318(19)$ & $0.0294(6)$ \\
\hline $\mathrm{O} 2$ & $0.3907(5)$ & $0.5919(3)$ & $0.66155(19)$ & $0.0298(6)$ \\
\hline $\mathrm{O} 3$ & $0.3627(5)$ & $0.9250(3)$ & $0.47327(18)$ & $0.0265(5)$ \\
\hline $\mathrm{O} 4$ & $0.3771(4)$ & $0.5007(3)$ & $0.14591(18)$ & $0.0265(5)$ \\
\hline O5 & $0.0097(4)$ & $-0.0028(3)$ & $-0.12980(18)$ & $0.0264(5)$ \\
\hline O6 & $0.0323(4)$ & $-0.3103(3)$ & $0.07559(18)$ & $0.0259(5)$ \\
\hline $\mathrm{C} 1$ & $-0.1767(6)$ & -0.2869 (4) & $0.0994(3)$ & $0.0245(7)$ \\
\hline $\mathrm{H} 1 \mathrm{~A}$ & -0.238920 & -0.369009 & 0.120950 & $0.029^{*}$ \\
\hline H1B & -0.275117 & -0.286764 & 0.042198 & $0.029^{*}$ \\
\hline $\mathrm{C} 2$ & $-0.1747(6)$ & $-0.1482(4)$ & $0.1741(2)$ & $0.0239(7)$ \\
\hline $\mathrm{C} 3$ & $-0.3613(6)$ & $-0.1340(4)$ & $0.2162(3)$ & $0.0266(7)$ \\
\hline $\mathrm{H} 3$ & -0.485939 & -0.207766 & 0.193390 & $0.032^{*}$ \\
\hline $\mathrm{C} 4$ & $-0.3703(6)$ & $-0.0156(4)$ & $0.2902(3)$ & $0.0267(7)$ \\
\hline $\mathrm{H} 4$ & -0.498316 & -0.007812 & 0.318459 & $0.032^{*}$ \\
\hline $\mathrm{C} 5$ & $-0.1889(6)$ & 0.0909 (4) & $0.3217(2)$ & $0.0222(6)$ \\
\hline C6 & $-0.0046(6)$ & $0.0796(4)$ & $0.2766(2)$ & $0.0220(6)$ \\
\hline C7 & $0.0060(6)$ & -0.0388 & $0.2035(2)$ & $0.0224(6)$ \\
\hline $\mathrm{H} 7$ & 0.132576 & -0.045426 & 0.174069 & $0.027^{*}$ \\
\hline $\mathrm{C} 8$ & $-0.1381(6)$ & $0.2194(4)$ & $0.4039(2)$ & $0.0225(6)$ \\
\hline C9 & $0.0774(6)$ & $0.2848(4)$ & $0.4097(2)$ & $0.0244(7)$ \\
\hline $\mathrm{C} 10$ & $0.1658(6)$ & $0.2076(4)$ & $0.3267(2)$ & $0.0231(7)$ \\
\hline $\mathrm{C} 11$ & $-0.2608(6)$ & $0.2752(4)$ & $0.4711(3)$ & $0.0261(7)$ \\
\hline H11 & -0.410474 & 0.235645 & 0.465987 & $0.031^{*}$ \\
\hline $\mathrm{C} 12$ & $-0.1588(7)$ & $0.3914(4)$ & $0.5467(3)$ & $0.0278(7)$ \\
\hline H12 & -0.242323 & 0.432694 & 0.592837 & $0.033^{*}$ \\
\hline $\mathrm{C} 13$ & $0.0608(6)$ & $0.4491(4)$ & $0.5571(2)$ & $0.0250(7)$ \\
\hline $\mathrm{C} 14$ & $0.1794(6)$ & $0.3979(4)$ & 0.4864 (3) & $0.0244(7)$ \\
\hline H14 & 0.327603 & 0.439670 & 0.490436 & $0.029^{*}$ \\
\hline $\mathrm{C} 15$ & $0.1603(7)$ & $0.5576(4)$ & 0.6484 (3) & $0.0290(8)$ \\
\hline $\mathrm{H} 15 \mathrm{~A}$ & 0.097362 & 0.647655 & 0.652418 & $0.035^{*}$ \\
\hline H15B & 0.118770 & 0.519599 & 0.700067 & $0.035^{*}$ \\
\hline C16 & $0.4764(6)$ & $0.7076(4)$ & $0.6331(2)$ & $0.0251(7)$ \\
\hline $\mathrm{C} 17$ & $0.6890(7)$ & 0.7711 & 0.6749 (3) & $0.0298(8)$ \\
\hline H17 & 0.766706 & 0.736390 & 0.720920 & $0.036^{*}$ \\
\hline $\mathrm{C} 18$ & $0.7850(7)$ & $0.8861(5)$ & 0.6479 (3) & $0.0316(8)$ \\
\hline
\end{tabular}




\begin{tabular}{|c|c|c|c|c|}
\hline H18 & 0.930083 & 0.931503 & 0.676396 & $0.038^{*}$ \\
\hline C19 & $0.6746(6)$ & 0.9367 (4) & $0.5802(3)$ & $0.0297(8)$ \\
\hline H19 & 0.743820 & 1.015419 & 0.561995 & $0.036^{*}$ \\
\hline $\mathrm{C} 20$ & $0.4627(6)$ & 0.8717 (4) & $0.5394(2)$ & $0.0245(7)$ \\
\hline $\mathrm{C} 21$ & $0.3627(6)$ & 0.7559 (4) & $0.5661(2)$ & $0.0256(7)$ \\
\hline $\mathrm{H} 21$ & 0.217055 & 0.710835 & 0.538118 & $0.031 *$ \\
\hline $\mathrm{C} 22$ & $0.1320(6)$ & $0.8832(4)$ & $0.4482(3)$ & $0.0260(7)$ \\
\hline $\mathrm{H} 22 \mathrm{~A}$ & 0.071510 & 0.954612 & 0.419840 & $0.031^{*}$ \\
\hline H22B & 0.068110 & 0.886179 & 0.505817 & $0.031 *$ \\
\hline $\mathrm{C} 23$ & $0.0619(6)$ & 0.7348 (4) & $0.3806(2)$ & $0.0231(7)$ \\
\hline $\mathrm{C} 24$ & $0.1890(6)$ & 0.6774 (4) & $0.3150(2)$ & $0.0242(7)$ \\
\hline $\mathrm{H} 24$ & 0.329679 & 0.727598 & 0.314978 & $0.029^{*}$ \\
\hline $\mathrm{C} 25$ & $0.1081(6)$ & $0.5461(4)$ & $0.2498(2)$ & $0.0220(6)$ \\
\hline $\mathrm{C} 26$ & $-0.0988(6)$ & 0.4699 (4) & $0.2487(2)$ & $0.0222(6)$ \\
\hline $\mathrm{C} 27$ & $-0.2240(6)$ & $0.5237(4)$ & $0.3154(3)$ & $0.0260(7)$ \\
\hline $\mathrm{H} 27$ & -0.362640 & 0.471796 & 0.316539 & $0.031 *$ \\
\hline $\mathrm{C} 28$ & $-0.1408(6)$ & $0.6572(4)$ & $0.3816(2)$ & $0.0250(7)$ \\
\hline $\mathrm{H} 28$ & -0.224639 & 0.695843 & 0.428392 & $0.030^{*}$ \\
\hline $\mathrm{C} 29$ & $0.2013(6)$ & $0.4666(4)$ & $0.1678(2)$ & $0.0230(6)$ \\
\hline $\mathrm{C} 30$ & $0.0310(6)$ & 0.3407 (4) & $0.1173(2)$ & $0.0236(7)$ \\
\hline $\mathrm{C} 31$ & $-0.1468(6)$ & 0.3419 (4) & $0.1661(2)$ & $0.0222(6)$ \\
\hline $\mathrm{C} 32$ & $0.0253(6)$ & $0.2392(4)$ & $0.0333(2)$ & $0.0232(6)$ \\
\hline H32 & 0.148081 & 0.238534 & 0.001502 & $0.028^{*}$ \\
\hline $\mathrm{C} 33$ & $-0.1620(6)$ & $0.1380(4)$ & $-0.0043(3)$ & $0.0243(7)$ \\
\hline C34 & $-0.3346(6)$ & 0.1355 (4) & $0.0463(3)$ & $0.0260(7)$ \\
\hline H34 & -0.459675 & 0.062432 & 0.021745 & $0.031^{*}$ \\
\hline $\mathrm{C} 35$ & $-0.3285(6)$ & $0.2373(4)$ & 0.1318 & $0.0258(7)$ \\
\hline H35 & -0.447445 & 0.234509 & 0.165682 & $0.031^{*}$ \\
\hline $\mathrm{C} 36$ & $-0.1881(6)$ & $0.0386(4)$ & $-0.1026(2)$ & $0.0261(7)$ \\
\hline H36A & -0.292015 & -0.049858 & -0.107536 & $0.031^{*}$ \\
\hline H36B & -0.253593 & 0.087410 & -0.147047 & $0.031^{*}$ \\
\hline $\mathrm{C} 37$ & $0.0872(6)$ & $-0.1085(4)$ & $-0.0968(2)$ & $0.0243(7)$ \\
\hline C38 & $0.2524(6)$ & $-0.1682(4)$ & -0.1384 & $0.0294(8)$ \\
\hline H38 & 0.305188 & -0.136765 & -0.187734 & $0.035^{*}$ \\
\hline C39 & $0.3404(6)$ & $-0.2748(4)$ & $-0.1071(3)$ & $0.0296(8)$ \\
\hline H39 & 0.454620 & -0.316585 & -0.135380 & $0.036^{*}$ \\
\hline $\mathrm{C} 40$ & $0.2643(6)$ & $-0.3216(4)$ & $-0.0352(3)$ & $0.0292(8)$ \\
\hline $\mathrm{H} 40$ & 0.325432 & -0.394801 & -0.014078 & $0.035^{*}$ \\
\hline $\mathrm{C} 41$ & $0.0986(6)$ & $-0.2601(4)$ & $0.0050(2)$ & $0.0250(7)$ \\
\hline $\mathrm{C} 42$ & $0.0074(6)$ & $-0.1549(4)$ & $-0.0254(3)$ & $0.0246(7)$ \\
\hline H42 & -0.108662 & -0.114593 & 0.002177 & $0.030^{*}$ \\
\hline $\mathrm{Cl1}$ & $0.2053(2)$ & $0.17453(13)$ & $0.65929(9)$ & $0.0542(4)$ \\
\hline $\mathrm{C} 43$ & $0.4128(7)$ & 0.2615 (4) & $0.7525(3)$ & $0.0306(8)$ \\
\hline $\mathrm{C} 44$ & $0.3680(7)$ & $0.2937(5)$ & 0.8418 (3) & $0.0320(8)$ \\
\hline H44 & 0.224785 & 0.265769 & 0.853168 & $0.038^{*}$ \\
\hline $\mathrm{C} 45$ & $0.5314(8)$ & $0.3668(5)$ & $0.9151(3)$ & $0.0348(9)$ \\
\hline $\mathrm{H} 45$ & 0.502082 & 0.389116 & 0.977568 & $0.042 *$ \\
\hline C46 & $0.7375(8)$ & $0.4077(5)$ & $0.8978(3)$ & $0.0406(10)$ \\
\hline
\end{tabular}




\begin{tabular}{lllll} 
H46 & 0.850033 & 0.460205 & 0.948342 & $0.049^{*}$ \\
C47 & $0.7812(9)$ & $0.3732(6)$ & $0.8083(4)$ & $0.0484(12)$ \\
H47 & 0.924417 & 0.401211 & 0.796899 & $0.058^{*}$ \\
C48 & $0.6189(9)$ & $0.2979(5)$ & $0.7341(3)$ & $0.0418(11)$ \\
H48 & 0.649317 & 0.271929 & 0.671735 & $0.050^{*}$ \\
\hline
\end{tabular}

Atomic displacement parameters $\left(\hat{A}^{2}\right)$

\begin{tabular}{|c|c|c|c|c|c|c|}
\hline & $U^{11}$ & $U^{22}$ & $U^{33}$ & $U^{12}$ & $U^{13}$ & $U^{23}$ \\
\hline $\mathrm{O} 1$ & $0.0216(13)$ & $0.0295(14)$ & $0.0336(14)$ & $-0.0023(10)$ & $0.0080(10)$ & $0.0040(11)$ \\
\hline $\mathrm{O} 2$ & $0.0340(15)$ & $0.0216(12)$ & $0.0304(13)$ & $-0.0017(10)$ & $0.0013(11)$ & $0.0058(10)$ \\
\hline $\mathrm{O} 3$ & $0.0278(14)$ & $0.0216(12)$ & $0.0279(13)$ & $0.0000(10)$ & $0.0036(10)$ & $0.0051(9)$ \\
\hline $\mathrm{O} 4$ & $0.0219(13)$ & $0.0244(13)$ & $0.0315(13)$ & $0.0004(10)$ & $0.0054(10)$ & $0.0058(10)$ \\
\hline O5 & $0.0267(14)$ & $0.0236(12)$ & $0.0270(12)$ & $-0.0006(10)$ & $0.0049(10)$ & $0.0057(10)$ \\
\hline O6 & $0.0241(13)$ & $0.0235(12)$ & $0.0300(13)$ & $0.0042(10)$ & $0.0055(10)$ & $0.0069(10)$ \\
\hline $\mathrm{C} 1$ & $0.0231(17)$ & $0.0225(16)$ & $0.0256(16)$ & $-0.0001(12)$ & $0.0047(12)$ & $0.0043(12)$ \\
\hline $\mathrm{C} 2$ & $0.0216(17)$ & $0.0219(16)$ & $0.0274(17)$ & 0.0009 (12) & $0.0033(13)$ & $0.0072(12)$ \\
\hline $\mathrm{C} 3$ & $0.0193(16)$ & $0.0246(17)$ & $0.0348(18)$ & $-0.0007(13)$ & $0.0064(13)$ & $0.0077(14)$ \\
\hline $\mathrm{C} 4$ & $0.0204(16)$ & $0.0256(17)$ & $0.0326(18)$ & $-0.0005(13)$ & 0.0067 (13) & $0.0064(14)$ \\
\hline $\mathrm{C} 5$ & $0.0227(17)$ & $0.0215(16)$ & $0.0219(15)$ & $0.0037(12)$ & $0.0035(12)$ & $0.0052(12)$ \\
\hline C6 & $0.0202(16)$ & $0.0233(16)$ & $0.0227(15)$ & $0.0016(12)$ & $0.0042(12)$ & $0.0074(12)$ \\
\hline $\mathrm{C} 7$ & 0.0210 & $0.0221(16)$ & $0.0232(15)$ & $0.0006(12)$ & $0.0052(12)$ & $0.0053(12)$ \\
\hline $\mathrm{C} 8$ & $0.0222(16)$ & $0.0214(16)$ & $0.0233(15)$ & 0.0009 (12) & $0.0072(12)$ & $0.0048(12)$ \\
\hline $\mathrm{C} 9$ & $0.0242(17)$ & $0.0220(16)$ & $0.0262(17)$ & 0.0018 (13) & $0.0055(13)$ & $0.0056(13)$ \\
\hline $\mathrm{C} 10$ & $0.0206(16)$ & $0.0224(16)$ & $0.0245(16)$ & $0.0021(12)$ & $0.0032(12)$ & $0.0044(12)$ \\
\hline $\mathrm{C} 11$ & $0.0225(17)$ & $0.0262(18)$ & $0.0282(17)$ & $0.0003(13)$ & $0.0065(13)$ & 0.0058 (13) \\
\hline $\mathrm{C} 12$ & 0.0289 (19) & $0.0269(18)$ & $0.0275(17)$ & $0.0025(14)$ & $0.0083(14)$ & $0.0067(14)$ \\
\hline $\mathrm{C} 13$ & $0.0271(18)$ & $0.0231(17)$ & $0.0237(16)$ & $-0.0005(13)$ & $0.0044(13)$ & $0.0064(13)$ \\
\hline $\mathrm{C} 14$ & $0.0258(18)$ & 0.0199 (16) & $0.0263(16)$ & 0.0007 (13) & $0.0040(13)$ & $0.0061(12)$ \\
\hline C15 & $0.032(2)$ & $0.0248(17)$ & 0.0268 (18) & $-0.0030(14)$ & $0.0045(14)$ & $0.0051(14)$ \\
\hline $\mathrm{C} 16$ & $0.0253(17)$ & $0.0234(16)$ & $0.0229(16)$ & $0.0005(13)$ & $0.0049(13)$ & $0.0009(12)$ \\
\hline C17 & $0.0252(18)$ & $0.032(2)$ & $0.0271(17)$ & $0.0011(14)$ & $0.0012(13)$ & $0.0030(14)$ \\
\hline $\mathrm{C} 18$ & $0.0242(18)$ & $0.035(2)$ & $0.0291(18)$ & $-0.0017(14)$ & $0.0027(14)$ & $0.0006(15)$ \\
\hline C19 & $0.0227(18)$ & $0.0254(18)$ & 0.0348 (19) & $-0.0057(13)$ & $0.0051(14)$ & $0.0019(14)$ \\
\hline $\mathrm{C} 20$ & $0.0242(17)$ & $0.0224(16)$ & $0.0252(16)$ & $0.0018(12)$ & $0.0055(12)$ & $0.0041(12)$ \\
\hline $\mathrm{C} 21$ & $0.0254(17)$ & $0.0224(16)$ & $0.0243(16)$ & $-0.0028(13)$ & $0.0027(12)$ & $0.0022(12)$ \\
\hline $\mathrm{C} 22$ & 0.0239 (17) & 0.0257 (17) & $0.0255(16)$ & $0.0027(13)$ & $0.0027(13)$ & $0.0031(13)$ \\
\hline $\mathrm{C} 23$ & $0.0229(17)$ & $0.0220(16)$ & $0.0240(16)$ & $0.0032(13)$ & $0.0021(12)$ & $0.0067(12)$ \\
\hline $\mathrm{C} 24$ & $0.0222(17)$ & $0.0242(16)$ & $0.0235(16)$ & $-0.0005(13)$ & $0.0045(12)$ & $0.0036(12)$ \\
\hline $\mathrm{C} 25$ & $0.0189(16)$ & $0.0238(16)$ & $0.0227(15)$ & $0.0011(12)$ & $0.0039(12)$ & $0.0062(12)$ \\
\hline $\mathrm{C} 26$ & $0.0189(15)$ & $0.0236(16)$ & $0.0207(15)$ & $0.0000(12)$ & $0.0018(11)$ & $0.0024(12)$ \\
\hline $\mathrm{C} 27$ & $0.0202(17)$ & $0.0284(18)$ & $0.0286(17)$ & $0.0017(13)$ & $0.0048(13)$ & $0.0071(14)$ \\
\hline $\mathrm{C} 28$ & $0.0225(17)$ & $0.0271(17)$ & $0.0232(16)$ & $0.0021(13)$ & $0.0036(13)$ & $0.0041(13)$ \\
\hline $\mathrm{C} 29$ & $0.0207(16)$ & $0.0219(15)$ & $0.0249(16)$ & $0.0014(12)$ & $0.0039(12)$ & $0.0051(12)$ \\
\hline $\mathrm{C} 30$ & $0.0193(16)$ & $0.0245(17)$ & $0.0259(16)$ & $0.0006(13)$ & $0.0027(12)$ & $0.0069(13)$ \\
\hline $\mathrm{C} 31$ & $0.0214(16)$ & $0.0218(15)$ & $0.0238(15)$ & $0.0027(12)$ & $0.0039(12)$ & $0.0073(12)$ \\
\hline $\mathrm{C} 32$ & $0.0212(16)$ & $0.0238(16)$ & $0.0245(16)$ & $0.0021(12)$ & $0.0043(12)$ & 0.0069 (13) \\
\hline C33 & $0.0228(17)$ & $0.0223(16)$ & $0.0273(16)$ & $0.0005(12)$ & $0.0025(13)$ & $0.0083(13)$ \\
\hline
\end{tabular}


supporting information

\begin{tabular}{lllllll} 
C34 & $0.0194(16)$ & $0.0245(17)$ & $0.0326(18)$ & $-0.0022(12)$ & $0.0013(13)$ & $0.0092(14)$ \\
C35 & $0.0196(16)$ & $0.0274(17)$ & $0.0302(17)$ & $-0.0004(13)$ & $0.0043(13)$ & $0.0095(13)$ \\
C36 & $0.0274(18)$ & $0.0229(16)$ & $0.0246(16)$ & $0.0010(13)$ & $0.0028(13)$ & $0.0031(12)$ \\
C37 & $0.0223(17)$ & $0.0241(16)$ & $0.0221(16)$ & $-0.0011(13)$ & $0.0009(12)$ & $0.0024(12)$ \\
C38 & $0.0209(17)$ & $0.0311(19)$ & $0.0293(18)$ & $-0.0044(14)$ & $0.0036(13)$ & $0.0008(14)$ \\
C39 & $0.0199(17)$ & $0.035(2)$ & $0.0293(18)$ & $0.0029(14)$ & $0.0072(13)$ & $0.0002(14)$ \\
C40 & $0.0198(17)$ & $0.0303(19)$ & $0.0333(19)$ & $0.0033(14)$ & $0.0017(14)$ & $0.0032(15)$ \\
C41 & $0.0210(16)$ & $0.0232(16)$ & $0.0261(16)$ & $-0.0021(12)$ & $0.0025(12)$ & $0.0024(13)$ \\
C42 & $0.0220(17)$ & $0.0218(16)$ & $0.0278(16)$ & $0.0012(12)$ & $0.0051(12)$ & $0.0036(12)$ \\
C11 & $0.0703(9)$ & $0.0375(6)$ & $0.0415(6)$ & $-0.0101(5)$ & $-0.0137(5)$ & $0.0074(5)$ \\
C43 & $0.035(2)$ & $0.0242(18)$ & $0.0315(19)$ & $0.0030(15)$ & $0.0041(15)$ & $0.0075(14)$ \\
C44 & $0.028(2)$ & $0.036(2)$ & $0.0318(19)$ & $0.0023(15)$ & $0.0064(15)$ & $0.0088(15)$ \\
C45 & $0.040(2)$ & $0.034(2)$ & $0.033(2)$ & $0.0101(17)$ & $0.0089(17)$ & $0.0093(16)$ \\
C46 & $0.037(2)$ & $0.0231(19)$ & $0.056(3)$ & $0.0001(16)$ & $-0.005(2)$ & $0.0093(18)$ \\
C47 & $0.036(3)$ & $0.042(3)$ & $0.074(4)$ & $-0.001(2)$ & $0.014(2)$ & $0.028(3)$ \\
C48 & $0.053(3)$ & $0.038(2)$ & $0.042(2)$ & $0.004(2)$ & $0.024(2)$ & $0.0173(19)$ \\
& & & & & \\
\hline
\end{tabular}

Geometric parameters $\left(\stackrel{A}{A}{ }^{\circ}\right)$

\begin{tabular}{llll}
\hline $\mathrm{O} 1-\mathrm{C} 10$ & $1.207(4)$ & $\mathrm{C} 22-\mathrm{H} 22 \mathrm{~B}$ & 0.9900 \\
$\mathrm{O} 2-\mathrm{C} 15$ & $1.410(5)$ & $\mathrm{C} 22-\mathrm{C} 23$ & $1.502(5)$ \\
$\mathrm{O} 2-\mathrm{C} 16$ & $1.374(5)$ & $\mathrm{C} 23-\mathrm{C} 24$ & $1.383(5)$ \\
$\mathrm{O} 3-\mathrm{C} 20$ & $1.353(5)$ & $\mathrm{C} 23-\mathrm{C} 28$ & $1.393(5)$ \\
$\mathrm{O} 3-\mathrm{C} 22$ & $1.420(5)$ & $\mathrm{C} 24-\mathrm{H} 24$ & 0.9500 \\
$\mathrm{O} 4-\mathrm{C} 29$ & $1.214(4)$ & $\mathrm{C} 24-\mathrm{C} 25$ & $1.377(5)$ \\
$\mathrm{O} 5-\mathrm{C} 36$ & $1.415(5)$ & $\mathrm{C} 25-\mathrm{C} 26$ & $1.402(5)$ \\
$\mathrm{O} 5-\mathrm{C} 37$ & $1.362(4)$ & $\mathrm{C} 25-\mathrm{C} 29$ & $1.492(5)$ \\
$\mathrm{O} 6-\mathrm{C} 1$ & $1.420(4)$ & $\mathrm{C} 26-\mathrm{C} 27$ & $1.377(5)$ \\
$\mathrm{O} 6-\mathrm{C} 41$ & $1.363(4)$ & $\mathrm{C} 26-\mathrm{C} 31$ & $1.473(5)$ \\
$\mathrm{C} 1-\mathrm{H} 1 \mathrm{~A}$ & 0.9900 & $\mathrm{C} 27-\mathrm{H} 27$ & 0.9500 \\
$\mathrm{C} 1-\mathrm{H} 1 \mathrm{~B}$ & 0.9900 & $\mathrm{C} 27-\mathrm{C} 28$ & $1.401(5)$ \\
$\mathrm{C} 1-\mathrm{C} 2$ & $1.504(5)$ & $\mathrm{C} 28-\mathrm{H} 28$ & 0.9500 \\
$\mathrm{C} 2-\mathrm{C} 3$ & $1.393(5)$ & $\mathrm{C} 29-\mathrm{C} 30$ & $1.479(5)$ \\
$\mathrm{C} 2-\mathrm{C} 7$ & $1.389(5)$ & $\mathrm{C} 30-\mathrm{C} 31$ & $1.400(5)$ \\
$\mathrm{C} 3-\mathrm{H} 3$ & 0.9500 & $\mathrm{C} 30-\mathrm{C} 32$ & $1.371(5)$ \\
$\mathrm{C} 3-\mathrm{C} 4$ & $1.382(5)$ & $\mathrm{C} 31-\mathrm{C} 35$ & $1.370(5)$ \\
$\mathrm{C} 4-\mathrm{H} 4$ & 0.9500 & $\mathrm{C} 32-\mathrm{H} 32$ & 0.9500 \\
$\mathrm{C} 4-\mathrm{C} 5$ & $1.376(5)$ & $\mathrm{C} 32-\mathrm{C} 33$ & $1.382(5)$ \\
$\mathrm{C} 5-\mathrm{C} 6$ & $1.404(5)$ & $\mathrm{C} 33-\mathrm{C} 34$ & $1.392(5)$ \\
$\mathrm{C} 5-\mathrm{C} 8$ & $1.472(5)$ & $\mathrm{C} 33-\mathrm{C} 36$ & $1.509(5)$ \\
$\mathrm{C} 6-\mathrm{C} 7$ & $1.377(5)$ & $\mathrm{C} 34-\mathrm{H} 34$ & 0.9500 \\
$\mathrm{C} 6-\mathrm{C} 10$ & $1.491(5)$ & $\mathrm{C} 34-\mathrm{C} 35$ & $1.389(5)$ \\
$\mathrm{C} 7-\mathrm{H} 7$ & $\mathrm{C} 35-\mathrm{H} 35$ & 0.9500 \\
$\mathrm{C} 8-\mathrm{C} 9$ & 1.39500 & $\mathrm{C} 36-\mathrm{H} 36 \mathrm{~A}$ & 0.9900 \\
$\mathrm{C} 8-\mathrm{C} 11$ & $\mathrm{C} 36-\mathrm{H} 36 \mathrm{~B}$ & 0.9900 \\
$\mathrm{C} 9-\mathrm{C} 10$ & $\mathrm{C} 37-\mathrm{C} 38$ & $1.377(5)$ \\
$\mathrm{C} 9-\mathrm{C} 14$ & $\mathrm{C} 37-\mathrm{C} 42$ & 0.9500 \\
$\mathrm{C} 11-\mathrm{H} 11$ & $1.376(5)$ & $\mathrm{C} 38-\mathrm{H} 38$ &
\end{tabular}




\begin{tabular}{|c|c|c|c|}
\hline $\mathrm{C} 11-\mathrm{C} 12$ & $1.390(5)$ & $\mathrm{C} 38-\mathrm{C} 39$ & $1.384(6)$ \\
\hline $\mathrm{C} 12-\mathrm{H} 12$ & 0.9500 & C39-H39 & 0.9500 \\
\hline $\mathrm{C} 12-\mathrm{C} 13$ & $1.387(5)$ & $\mathrm{C} 39-\mathrm{C} 40$ & $1.387(6)$ \\
\hline $\mathrm{C} 13-\mathrm{C} 14$ & $1.386(5)$ & $\mathrm{C} 40-\mathrm{H} 40$ & 0.9500 \\
\hline $\mathrm{C} 13-\mathrm{C} 15$ & $1.495(5)$ & $\mathrm{C} 40-\mathrm{C} 41$ & $1.377(5)$ \\
\hline C14-H14 & 0.9500 & $\mathrm{C} 41-\mathrm{C} 42$ & $1.375(5)$ \\
\hline C15-H15A & 0.9900 & $\mathrm{C} 42-\mathrm{H} 42$ & 0.9500 \\
\hline C15-H15B & 0.9900 & $\mathrm{Cl1}-\mathrm{C} 43$ & $1.732(4)$ \\
\hline $\mathrm{C} 16-\mathrm{C} 17$ & $1.385(5)$ & $\mathrm{C} 43-\mathrm{C} 44$ & $1.362(5)$ \\
\hline $\mathrm{C} 16-\mathrm{C} 21$ & $1.372(5)$ & $\mathrm{C} 43-\mathrm{C} 48$ & $1.371(6)$ \\
\hline C17-H17 & 0.9500 & $\mathrm{C} 44-\mathrm{H} 44$ & 0.9500 \\
\hline $\mathrm{C} 17-\mathrm{C} 18$ & $1.378(6)$ & $\mathrm{C} 44-\mathrm{C} 45$ & $1.372(6)$ \\
\hline $\mathrm{C} 18-\mathrm{H} 18$ & 0.9500 & $\mathrm{C} 45-\mathrm{H} 45$ & 0.9500 \\
\hline $\mathrm{C} 18-\mathrm{C} 19$ & $1.383(6)$ & $\mathrm{C} 45-\mathrm{C} 46$ & $1.373(7)$ \\
\hline C19-H19 & 0.9500 & $\mathrm{C} 46-\mathrm{H} 46$ & 0.9500 \\
\hline $\mathrm{C} 19-\mathrm{C} 20$ & $1.382(5)$ & $\mathrm{C} 46-\mathrm{C} 47$ & $1.364(7)$ \\
\hline $\mathrm{C} 20-\mathrm{C} 21$ & $1.392(5)$ & C47-H47 & 0.9500 \\
\hline $\mathrm{C} 21-\mathrm{H} 21$ & 0.9500 & $\mathrm{C} 47-\mathrm{C} 48$ & $1.381(7)$ \\
\hline $\mathrm{C} 22-\mathrm{H} 22 \mathrm{~A}$ & 0.9900 & $\mathrm{C} 48-\mathrm{H} 48$ & 0.9500 \\
\hline $\mathrm{C} 16-\mathrm{O} 2-\mathrm{C} 15$ & $117.1(3)$ & $\mathrm{C} 24-\mathrm{C} 23-\mathrm{C} 28$ & $119.8(3)$ \\
\hline $\mathrm{C} 20-\mathrm{O} 3-\mathrm{C} 22$ & $116.5(3)$ & $\mathrm{C} 28-\mathrm{C} 23-\mathrm{C} 22$ & $118.7(3)$ \\
\hline $\mathrm{C} 37-\mathrm{O} 5-\mathrm{C} 36$ & $116.8(3)$ & $\mathrm{C} 23-\mathrm{C} 24-\mathrm{H} 24$ & 120.6 \\
\hline $\mathrm{C} 41-\mathrm{O} 6-\mathrm{C} 1$ & $118.1(3)$ & $\mathrm{C} 25-\mathrm{C} 24-\mathrm{C} 23$ & $118.8(3)$ \\
\hline $\mathrm{O} 6-\mathrm{C} 1-\mathrm{H} 1 \mathrm{~A}$ & 108.6 & $\mathrm{C} 25-\mathrm{C} 24-\mathrm{H} 24$ & 120.6 \\
\hline $\mathrm{O} 6-\mathrm{C} 1-\mathrm{H} 1 \mathrm{~B}$ & 108.6 & $\mathrm{C} 24-\mathrm{C} 25-\mathrm{C} 26$ & $121.5(3)$ \\
\hline $\mathrm{O} 6-\mathrm{C} 1-\mathrm{C} 2$ & $114.5(3)$ & $\mathrm{C} 24-\mathrm{C} 25-\mathrm{C} 29$ & $129.7(3)$ \\
\hline $\mathrm{H} 1 \mathrm{~A}-\mathrm{C} 1-\mathrm{H} 1 \mathrm{~B}$ & 107.6 & $\mathrm{C} 26-\mathrm{C} 25-\mathrm{C} 29$ & $108.5(3)$ \\
\hline $\mathrm{C} 2-\mathrm{C} 1-\mathrm{H} 1 \mathrm{~A}$ & 108.6 & $\mathrm{C} 25-\mathrm{C} 26-\mathrm{C} 31$ & $108.6(3)$ \\
\hline $\mathrm{C} 2-\mathrm{C} 1-\mathrm{H} 1 \mathrm{~B}$ & 108.6 & $\mathrm{C} 27-\mathrm{C} 26-\mathrm{C} 25$ & $120.2(3)$ \\
\hline $\mathrm{C} 3-\mathrm{C} 2-\mathrm{C} 1$ & $117.3(3)$ & $\mathrm{C} 27-\mathrm{C} 26-\mathrm{C} 31$ & $131.1(3)$ \\
\hline $\mathrm{C} 7-\mathrm{C} 2-\mathrm{C} 1$ & $122.5(3)$ & $\mathrm{C} 26-\mathrm{C} 27-\mathrm{H} 27$ & 121.0 \\
\hline $\mathrm{C} 7-\mathrm{C} 2-\mathrm{C} 3$ & $120.2(3)$ & $\mathrm{C} 26-\mathrm{C} 27-\mathrm{C} 28$ & $118.0(3)$ \\
\hline $\mathrm{C} 2-\mathrm{C} 3-\mathrm{H} 3$ & 119.1 & $\mathrm{C} 28-\mathrm{C} 27-\mathrm{H} 27$ & 121.0 \\
\hline $\mathrm{C} 4-\mathrm{C} 3-\mathrm{C} 2$ & $121.9(3)$ & $\mathrm{C} 23-\mathrm{C} 28-\mathrm{C} 27$ & $121.6(3)$ \\
\hline $\mathrm{C} 4-\mathrm{C} 3-\mathrm{H} 3$ & 119.1 & $\mathrm{C} 23-\mathrm{C} 28-\mathrm{H} 28$ & 119.2 \\
\hline $\mathrm{C} 3-\mathrm{C} 4-\mathrm{H} 4$ & 120.9 & $\mathrm{C} 27-\mathrm{C} 28-\mathrm{H} 28$ & 119.2 \\
\hline $\mathrm{C} 5-\mathrm{C} 4-\mathrm{C} 3$ & $118.3(3)$ & $\mathrm{O} 4-\mathrm{C} 29-\mathrm{C} 25$ & $127.1(3)$ \\
\hline $\mathrm{C} 5-\mathrm{C} 4-\mathrm{H} 4$ & 120.9 & $\mathrm{O} 4-\mathrm{C} 29-\mathrm{C} 30$ & $127.5(3)$ \\
\hline $\mathrm{C} 4-\mathrm{C} 5-\mathrm{C} 6$ & $119.9(3)$ & $\mathrm{C} 30-\mathrm{C} 29-\mathrm{C} 25$ & $105.3(3)$ \\
\hline $\mathrm{C} 4-\mathrm{C} 5-\mathrm{C} 8$ & $131.2(3)$ & $\mathrm{C} 31-\mathrm{C} 30-\mathrm{C} 29$ & $109.1(3)$ \\
\hline $\mathrm{C} 6-\mathrm{C} 5-\mathrm{C} 8$ & $108.7(3)$ & $\mathrm{C} 32-\mathrm{C} 30-\mathrm{C} 29$ & $129.5(3)$ \\
\hline $\mathrm{C} 5-\mathrm{C} 6-\mathrm{C} 10$ & $107.9(3)$ & $\mathrm{C} 32-\mathrm{C} 30-\mathrm{C} 31$ & $121.3(3)$ \\
\hline $\mathrm{C} 7-\mathrm{C} 6-\mathrm{C} 5$ & $122.1(3)$ & $\mathrm{C} 30-\mathrm{C} 31-\mathrm{C} 26$ & $108.4(3)$ \\
\hline $\mathrm{C} 7-\mathrm{C} 6-\mathrm{C} 10$ & $129.9(3)$ & $\mathrm{C} 35-\mathrm{C} 31-\mathrm{C} 26$ & $131.4(3)$ \\
\hline $\mathrm{C} 2-\mathrm{C} 7-\mathrm{H} 7$ & 121.2 & $\mathrm{C} 35-\mathrm{C} 31-\mathrm{C} 30$ & $120.1(3)$ \\
\hline $\mathrm{C} 6-\mathrm{C} 7-\mathrm{C} 2$ & $117.7(3)$ & $\mathrm{C} 30-\mathrm{C} 32-\mathrm{H} 32$ & 120.5 \\
\hline $\mathrm{C} 6-\mathrm{C} 7-\mathrm{H} 7$ & 121.2 & $\mathrm{C} 30-\mathrm{C} 32-\mathrm{C} 33$ & $119.0(3)$ \\
\hline
\end{tabular}




\begin{tabular}{|c|c|c|c|}
\hline $\mathrm{C} 9-\mathrm{C} 8-\mathrm{C} 5$ & $108.5(3)$ & $\mathrm{C} 33-\mathrm{C} 32-\mathrm{H} 32$ & 120.5 \\
\hline $\mathrm{C} 11-\mathrm{C} 8-\mathrm{C} 5$ & $131.3(3)$ & $\mathrm{C} 32-\mathrm{C} 33-\mathrm{C} 34$ & $119.5(3)$ \\
\hline $\mathrm{C} 11-\mathrm{C} 8-\mathrm{C} 9$ & $120.1(3)$ & $\mathrm{C} 32-\mathrm{C} 33-\mathrm{C} 36$ & $120.7(3)$ \\
\hline $\mathrm{C} 8-\mathrm{C} 9-\mathrm{C} 10$ & $108.7(3)$ & $\mathrm{C} 34-\mathrm{C} 33-\mathrm{C} 36$ & $119.7(3)$ \\
\hline $\mathrm{C} 14-\mathrm{C} 9-\mathrm{C} 8$ & $121.4(3)$ & $\mathrm{C} 33-\mathrm{C} 34-\mathrm{H} 34$ & 119.2 \\
\hline $\mathrm{C} 14-\mathrm{C} 9-\mathrm{C} 10$ & $129.8(3)$ & $\mathrm{C} 35-\mathrm{C} 34-\mathrm{C} 33$ & $121.6(3)$ \\
\hline $\mathrm{O} 1-\mathrm{C} 10-\mathrm{C} 6$ & $126.6(3)$ & $\mathrm{C} 35-\mathrm{C} 34-\mathrm{H} 34$ & 119.2 \\
\hline $\mathrm{O} 1-\mathrm{C} 10-\mathrm{C} 9$ & $127.7(3)$ & $\mathrm{C} 31-\mathrm{C} 35-\mathrm{C} 34$ & $118.4(3)$ \\
\hline $\mathrm{C} 9-\mathrm{C} 10-\mathrm{C} 6$ & $105.7(3)$ & $\mathrm{C} 31-\mathrm{C} 35-\mathrm{H} 35$ & 120.8 \\
\hline $\mathrm{C} 8-\mathrm{C} 11-\mathrm{H} 11$ & 121.1 & $\mathrm{C} 34-\mathrm{C} 35-\mathrm{H} 35$ & 120.8 \\
\hline $\mathrm{C} 8-\mathrm{C} 11-\mathrm{C} 12$ & $117.9(3)$ & $\mathrm{O} 5-\mathrm{C} 36-\mathrm{C} 33$ & $114.3(3)$ \\
\hline $\mathrm{C} 12-\mathrm{C} 11-\mathrm{H} 11$ & 121.1 & $\mathrm{O} 5-\mathrm{C} 36-\mathrm{H} 36 \mathrm{~A}$ & 108.7 \\
\hline $\mathrm{C} 11-\mathrm{C} 12-\mathrm{H} 12$ & 118.9 & $\mathrm{O} 5-\mathrm{C} 36-\mathrm{H} 36 \mathrm{~B}$ & 108.7 \\
\hline $\mathrm{C} 13-\mathrm{C} 12-\mathrm{C} 11$ & $122.3(4)$ & $\mathrm{C} 33-\mathrm{C} 36-\mathrm{H} 36 \mathrm{~A}$ & 108.7 \\
\hline $\mathrm{C} 13-\mathrm{C} 12-\mathrm{H} 12$ & 118.9 & $\mathrm{C} 33-\mathrm{C} 36-\mathrm{H} 36 \mathrm{~B}$ & 108.7 \\
\hline $\mathrm{C} 12-\mathrm{C} 13-\mathrm{C} 15$ & $117.1(3)$ & $\mathrm{H} 36 \mathrm{~A}-\mathrm{C} 36-\mathrm{H} 36 \mathrm{~B}$ & 107.6 \\
\hline $\mathrm{C} 14-\mathrm{C} 13-\mathrm{C} 12$ & $119.3(3)$ & $\mathrm{O} 5-\mathrm{C} 37-\mathrm{C} 38$ & $116.1(3)$ \\
\hline $\mathrm{C} 14-\mathrm{C} 13-\mathrm{C} 15$ & $123.4(3)$ & $\mathrm{O} 5-\mathrm{C} 37-\mathrm{C} 42$ & $123.0(3)$ \\
\hline $\mathrm{C} 9-\mathrm{C} 14-\mathrm{C} 13$ & $118.6(3)$ & $\mathrm{C} 38-\mathrm{C} 37-\mathrm{C} 42$ & $120.9(4)$ \\
\hline C9-C14-H14 & 120.7 & $\mathrm{C} 37-\mathrm{C} 38-\mathrm{H} 38$ & 120.5 \\
\hline $\mathrm{C} 13-\mathrm{C} 14-\mathrm{H} 14$ & 120.7 & $\mathrm{C} 37-\mathrm{C} 38-\mathrm{C} 39$ & $118.9(3)$ \\
\hline $\mathrm{O} 2-\mathrm{C} 15-\mathrm{C} 13$ & $114.4(3)$ & $\mathrm{C} 39-\mathrm{C} 38-\mathrm{H} 38$ & 120.5 \\
\hline $\mathrm{O} 2-\mathrm{C} 15-\mathrm{H} 15 \mathrm{~A}$ & 108.7 & $\mathrm{C} 38-\mathrm{C} 39-\mathrm{H} 39$ & 119.5 \\
\hline $\mathrm{O} 2-\mathrm{C} 15-\mathrm{H} 15 \mathrm{~B}$ & 108.7 & $\mathrm{C} 38-\mathrm{C} 39-\mathrm{C} 40$ & $121.0(4)$ \\
\hline $\mathrm{C} 13-\mathrm{C} 15-\mathrm{H} 15 \mathrm{~A}$ & 108.7 & $\mathrm{C} 40-\mathrm{C} 39-\mathrm{H} 39$ & 119.5 \\
\hline $\mathrm{C} 13-\mathrm{C} 15-\mathrm{H} 15 \mathrm{~B}$ & 108.7 & $\mathrm{C} 39-\mathrm{C} 40-\mathrm{H} 40$ & 120.6 \\
\hline $\mathrm{H} 15 \mathrm{~A}-\mathrm{C} 15-\mathrm{H} 15 \mathrm{~B}$ & 107.6 & $\mathrm{C} 41-\mathrm{C} 40-\mathrm{C} 39$ & $118.8(4)$ \\
\hline $\mathrm{O} 2-\mathrm{C} 16-\mathrm{C} 17$ & $115.7(3)$ & $\mathrm{C} 41-\mathrm{C} 40-\mathrm{H} 40$ & 120.6 \\
\hline $\mathrm{C} 21-\mathrm{C} 16-\mathrm{O} 2$ & $122.8(3)$ & $\mathrm{O} 6-\mathrm{C} 41-\mathrm{C} 40$ & $115.9(3)$ \\
\hline $\mathrm{C} 21-\mathrm{C} 16-\mathrm{C} 17$ & $121.5(3)$ & $\mathrm{O} 6-\mathrm{C} 41-\mathrm{C} 42$ & $122.9(3)$ \\
\hline $\mathrm{C} 16-\mathrm{C} 17-\mathrm{H} 17$ & 120.9 & $\mathrm{C} 42-\mathrm{C} 41-\mathrm{C} 40$ & $121.2(3)$ \\
\hline $\mathrm{C} 18-\mathrm{C} 17-\mathrm{C} 16$ & $118.3(4)$ & $\mathrm{C} 37-\mathrm{C} 42-\mathrm{H} 42$ & 120.5 \\
\hline $\mathrm{C} 18-\mathrm{C} 17-\mathrm{H} 17$ & 120.9 & $\mathrm{C} 41-\mathrm{C} 42-\mathrm{C} 37$ & $119.1(3)$ \\
\hline $\mathrm{C} 17-\mathrm{C} 18-\mathrm{H} 18$ & 119.3 & $\mathrm{C} 41-\mathrm{C} 42-\mathrm{H} 42$ & 120.5 \\
\hline $\mathrm{C} 17-\mathrm{C} 18-\mathrm{C} 19$ & $121.5(4)$ & $\mathrm{C} 44-\mathrm{C} 43-\mathrm{Cl} 1$ & $119.8(3)$ \\
\hline $\mathrm{C} 19-\mathrm{C} 18-\mathrm{H} 18$ & 119.3 & $\mathrm{C} 44-\mathrm{C} 43-\mathrm{C} 48$ & $121.6(4)$ \\
\hline $\mathrm{C} 18-\mathrm{C} 19-\mathrm{H} 19$ & 120.3 & $\mathrm{C} 48-\mathrm{C} 43-\mathrm{Cl} 1$ & $118.6(3)$ \\
\hline $\mathrm{C} 20-\mathrm{C} 19-\mathrm{C} 18$ & $119.3(3)$ & $\mathrm{C} 43-\mathrm{C} 44-\mathrm{H} 44$ & 120.3 \\
\hline $\mathrm{C} 20-\mathrm{C} 19-\mathrm{H} 19$ & 120.3 & $\mathrm{C} 43-\mathrm{C} 44-\mathrm{C} 45$ & $119.5(4)$ \\
\hline $\mathrm{O} 3-\mathrm{C} 20-\mathrm{C} 19$ & $116.9(3)$ & $\mathrm{C} 45-\mathrm{C} 44-\mathrm{H} 44$ & 120.3 \\
\hline $\mathrm{O} 3-\mathrm{C} 20-\mathrm{C} 21$ & $123.1(3)$ & $\mathrm{C} 44-\mathrm{C} 45-\mathrm{H} 45$ & 120.1 \\
\hline $\mathrm{C} 19-\mathrm{C} 20-\mathrm{C} 21$ & $120.0(4)$ & $\mathrm{C} 44-\mathrm{C} 45-\mathrm{C} 46$ & $119.8(4)$ \\
\hline $\mathrm{C} 16-\mathrm{C} 21-\mathrm{C} 20$ & $119.4(3)$ & $\mathrm{C} 46-\mathrm{C} 45-\mathrm{H} 45$ & 120.1 \\
\hline $\mathrm{C} 16-\mathrm{C} 21-\mathrm{H} 21$ & 120.3 & $\mathrm{C} 45-\mathrm{C} 46-\mathrm{H} 46$ & 119.9 \\
\hline $\mathrm{C} 20-\mathrm{C} 21-\mathrm{H} 21$ & 120.3 & $\mathrm{C} 47-\mathrm{C} 46-\mathrm{C} 45$ & $120.2(4)$ \\
\hline $\mathrm{O} 3-\mathrm{C} 22-\mathrm{H} 22 \mathrm{~A}$ & 108.7 & $\mathrm{C} 47-\mathrm{C} 46-\mathrm{H} 46$ & 119.9 \\
\hline $\mathrm{O} 3-\mathrm{C} 22-\mathrm{H} 22 \mathrm{~B}$ & 108.7 & $\mathrm{C} 46-\mathrm{C} 47-\mathrm{H} 47$ & 119.7 \\
\hline $\mathrm{O} 3-\mathrm{C} 22-\mathrm{C} 23$ & $114.1(3)$ & $\mathrm{C} 46-\mathrm{C} 47-\mathrm{C} 48$ & $120.6(4)$ \\
\hline
\end{tabular}




\begin{tabular}{|c|c|c|c|}
\hline $\mathrm{H} 22 \mathrm{~A}-\mathrm{C} 22-\mathrm{H} 22 \mathrm{~B}$ & 107.6 & $\mathrm{C} 48-\mathrm{C} 47-\mathrm{H} 47$ & 119.7 \\
\hline $\mathrm{C} 23-\mathrm{C} 22-\mathrm{H} 22 \mathrm{~A}$ & 108.7 & $\mathrm{C} 43-\mathrm{C} 48-\mathrm{C} 47$ & $118.3(4)$ \\
\hline $\mathrm{C} 23-\mathrm{C} 22-\mathrm{H} 22 \mathrm{~B}$ & 108.7 & $\mathrm{C} 43-\mathrm{C} 48-\mathrm{H} 48$ & 120.8 \\
\hline $\mathrm{C} 24-\mathrm{C} 23-\mathrm{C} 22$ & $121.4(3)$ & $\mathrm{C} 47-\mathrm{C} 48-\mathrm{H} 48$ & 120.8 \\
\hline $\mathrm{O} 2-\mathrm{C} 16-\mathrm{C} 17-\mathrm{C} 18$ & $178.9(3)$ & $\mathrm{C} 19-\mathrm{C} 20-\mathrm{C} 21-\mathrm{C} 16$ & $0.1(5)$ \\
\hline $\mathrm{O} 2-\mathrm{C} 16-\mathrm{C} 21-\mathrm{C} 20$ & $-178.5(3)$ & $\mathrm{C} 20-\mathrm{O} 3-\mathrm{C} 22-\mathrm{C} 23$ & $-80.0(4)$ \\
\hline $\mathrm{O} 3-\mathrm{C} 20-\mathrm{C} 21-\mathrm{C} 16$ & $179.4(3)$ & $\mathrm{C} 21-\mathrm{C} 16-\mathrm{C} 17-\mathrm{C} 18$ & $0.5(6)$ \\
\hline $\mathrm{O} 3-\mathrm{C} 22-\mathrm{C} 23-\mathrm{C} 24$ & $-30.7(5)$ & $\mathrm{C} 22-\mathrm{O} 3-\mathrm{C} 20-\mathrm{C} 19$ & $-165.2(3)$ \\
\hline $\mathrm{O} 3-\mathrm{C} 22-\mathrm{C} 23-\mathrm{C} 28$ & $152.4(3)$ & $\mathrm{C} 22-\mathrm{O} 3-\mathrm{C} 20-\mathrm{C} 21$ & $15.4(5)$ \\
\hline $\mathrm{O} 4-\mathrm{C} 29-\mathrm{C} 30-\mathrm{C} 31$ & $-179.6(4)$ & $\mathrm{C} 22-\mathrm{C} 23-\mathrm{C} 24-\mathrm{C} 25$ & $-174.6(3)$ \\
\hline $\mathrm{O} 4-\mathrm{C} 29-\mathrm{C} 30-\mathrm{C} 32$ & $-3.9(7)$ & $\mathrm{C} 22-\mathrm{C} 23-\mathrm{C} 28-\mathrm{C} 27$ & $174.6(3)$ \\
\hline $\mathrm{O} 5-\mathrm{C} 37-\mathrm{C} 38-\mathrm{C} 39$ & $179.1(3)$ & $\mathrm{C} 23-\mathrm{C} 24-\mathrm{C} 25-\mathrm{C} 26$ & $-0.1(5)$ \\
\hline $\mathrm{O} 5-\mathrm{C} 37-\mathrm{C} 42-\mathrm{C} 41$ & $-178.6(3)$ & $\mathrm{C} 23-\mathrm{C} 24-\mathrm{C} 25-\mathrm{C} 29$ & $174.4(3)$ \\
\hline $\mathrm{O} 6-\mathrm{C} 1-\mathrm{C} 2-\mathrm{C} 3$ & $-165.4(3)$ & $\mathrm{C} 24-\mathrm{C} 23-\mathrm{C} 28-\mathrm{C} 27$ & $-2.3(5)$ \\
\hline $\mathrm{O} 6-\mathrm{C} 1-\mathrm{C} 2-\mathrm{C} 7$ & $12.6(5)$ & $\mathrm{C} 24-\mathrm{C} 25-\mathrm{C} 26-\mathrm{C} 27$ & $-1.9(5)$ \\
\hline $\mathrm{O} 6-\mathrm{C} 41-\mathrm{C} 42-\mathrm{C} 37$ & $179.0(3)$ & $\mathrm{C} 24-\mathrm{C} 25-\mathrm{C} 26-\mathrm{C} 31$ & $174.7(3)$ \\
\hline $\mathrm{C} 1-\mathrm{O} 6-\mathrm{C} 41-\mathrm{C} 40$ & $-160.6(3)$ & $\mathrm{C} 24-\mathrm{C} 25-\mathrm{C} 29-\mathrm{O} 4$ & $4.5(6)$ \\
\hline $\mathrm{C} 1-\mathrm{O} 6-\mathrm{C} 41-\mathrm{C} 42$ & $19.3(5)$ & $\mathrm{C} 24-\mathrm{C} 25-\mathrm{C} 29-\mathrm{C} 30$ & $-173.6(4)$ \\
\hline $\mathrm{C} 1-\mathrm{C} 2-\mathrm{C} 3-\mathrm{C} 4$ & $175.2(4)$ & $\mathrm{C} 25-\mathrm{C} 26-\mathrm{C} 27-\mathrm{C} 28$ & $1.8(5)$ \\
\hline $\mathrm{C} 1-\mathrm{C} 2-\mathrm{C} 7-\mathrm{C} 6$ & $-175.9(3)$ & $\mathrm{C} 25-\mathrm{C} 26-\mathrm{C} 31-\mathrm{C} 30$ & $0.0(4)$ \\
\hline $\mathrm{C} 2-\mathrm{C} 3-\mathrm{C} 4-\mathrm{C} 5$ & $0.5(6)$ & $\mathrm{C} 25-\mathrm{C} 26-\mathrm{C} 31-\mathrm{C} 35$ & $-177.1(4)$ \\
\hline $\mathrm{C} 3-\mathrm{C} 2-\mathrm{C} 7-\mathrm{C} 6$ & $2.1(5)$ & $\mathrm{C} 25-\mathrm{C} 29-\mathrm{C} 30-\mathrm{C} 31$ & $-1.5(4)$ \\
\hline $\mathrm{C} 3-\mathrm{C} 4-\mathrm{C} 5-\mathrm{C} 6$ & $2.3(5)$ & $\mathrm{C} 25-\mathrm{C} 29-\mathrm{C} 30-\mathrm{C} 32$ & $174.2(4)$ \\
\hline $\mathrm{C} 3-\mathrm{C} 4-\mathrm{C} 5-\mathrm{C} 8$ & $-172.2(4)$ & $\mathrm{C} 26-\mathrm{C} 25-\mathrm{C} 29-\mathrm{O} 4$ & $179.6(4)$ \\
\hline $\mathrm{C} 4-\mathrm{C} 5-\mathrm{C} 6-\mathrm{C} 7$ & $-3.1(5)$ & $\mathrm{C} 26-\mathrm{C} 25-\mathrm{C} 29-\mathrm{C} 30$ & $1.5(4)$ \\
\hline $\mathrm{C} 4-\mathrm{C} 5-\mathrm{C} 6-\mathrm{C} 10$ & $-179.0(3)$ & $\mathrm{C} 26-\mathrm{C} 27-\mathrm{C} 28-\mathrm{C} 23$ & $0.3(6)$ \\
\hline $\mathrm{C} 4-\mathrm{C} 5-\mathrm{C} 8-\mathrm{C} 9$ & $174.1(4)$ & $\mathrm{C} 26-\mathrm{C} 31-\mathrm{C} 35-\mathrm{C} 34$ & $174.0(4)$ \\
\hline $\mathrm{C} 4-\mathrm{C} 5-\mathrm{C} 8-\mathrm{C} 11$ & $-3.6(7)$ & $\mathrm{C} 27-\mathrm{C} 26-\mathrm{C} 31-\mathrm{C} 30$ & $176.1(4)$ \\
\hline $\mathrm{C} 5-\mathrm{C} 6-\mathrm{C} 7-\mathrm{C} 2$ & $0.8(5)$ & $\mathrm{C} 27-\mathrm{C} 26-\mathrm{C} 31-\mathrm{C} 35$ & $-1.0(7)$ \\
\hline $\mathrm{C} 5-\mathrm{C} 6-\mathrm{C} 10-\mathrm{O} 1$ & $-173.4(4)$ & $\mathrm{C} 28-\mathrm{C} 23-\mathrm{C} 24-\mathrm{C} 25$ & $2.2(5)$ \\
\hline $\mathrm{C} 5-\mathrm{C} 6-\mathrm{C} 10-\mathrm{C} 9$ & $6.1(4)$ & $\mathrm{C} 29-\mathrm{C} 25-\mathrm{C} 26-\mathrm{C} 27$ & $-177.5(3)$ \\
\hline $\mathrm{C} 5-\mathrm{C} 8-\mathrm{C} 9-\mathrm{C} 10$ & $4.8(4)$ & $\mathrm{C} 29-\mathrm{C} 25-\mathrm{C} 26-\mathrm{C} 31$ & $-0.9(4)$ \\
\hline $\mathrm{C} 5-\mathrm{C} 8-\mathrm{C} 9-\mathrm{C} 14$ & $-172.1(3)$ & $\mathrm{C} 29-\mathrm{C} 30-\mathrm{C} 31-\mathrm{C} 26$ & $1.0(4)$ \\
\hline $\mathrm{C} 5-\mathrm{C} 8-\mathrm{C} 11-\mathrm{C} 12$ & $173.4(4)$ & $\mathrm{C} 29-\mathrm{C} 30-\mathrm{C} 31-\mathrm{C} 35$ & $178.4(3)$ \\
\hline $\mathrm{C} 6-\mathrm{C} 5-\mathrm{C} 8-\mathrm{C} 9$ & $-0.9(4)$ & $\mathrm{C} 29-\mathrm{C} 30-\mathrm{C} 32-\mathrm{C} 33$ & $-174.1(4)$ \\
\hline $\mathrm{C} 6-\mathrm{C} 5-\mathrm{C} 8-\mathrm{C} 11$ & $-178.6(4)$ & $\mathrm{C} 30-\mathrm{C} 31-\mathrm{C} 35-\mathrm{C} 34$ & $-2.8(5)$ \\
\hline $\mathrm{C} 7-\mathrm{C} 2-\mathrm{C} 3-\mathrm{C} 4$ & $-2.9(5)$ & $\mathrm{C} 30-\mathrm{C} 32-\mathrm{C} 33-\mathrm{C} 34$ & $-4.0(5)$ \\
\hline $\mathrm{C} 7-\mathrm{C} 6-\mathrm{C} 10-\mathrm{O} 1$ & $11.1(6)$ & $\mathrm{C} 30-\mathrm{C} 32-\mathrm{C} 33-\mathrm{C} 36$ & $171.1(3)$ \\
\hline $\mathrm{C} 7-\mathrm{C} 6-\mathrm{C} 10-\mathrm{C} 9$ & $-169.4(4)$ & $\mathrm{C} 31-\mathrm{C} 26-\mathrm{C} 27-\mathrm{C} 28$ & $-173.9(4)$ \\
\hline $\mathrm{C} 8-\mathrm{C} 5-\mathrm{C} 6-\mathrm{C} 7$ & $172.6(3)$ & $\mathrm{C} 31-\mathrm{C} 30-\mathrm{C} 32-\mathrm{C} 33$ & $1.2(5)$ \\
\hline $\mathrm{C} 8-\mathrm{C} 5-\mathrm{C} 6-\mathrm{C} 10$ & $-3.3(4)$ & $\mathrm{C} 32-\mathrm{C} 30-\mathrm{C} 31-\mathrm{C} 26$ & $-175.2(3)$ \\
\hline $\mathrm{C} 8-\mathrm{C} 9-\mathrm{C} 10-\mathrm{O} 1$ & $172.7(4)$ & $\mathrm{C} 32-\mathrm{C} 30-\mathrm{C} 31-\mathrm{C} 35$ & $2.3(5)$ \\
\hline $\mathrm{C} 8-\mathrm{C} 9-\mathrm{C} 10-\mathrm{C} 6$ & $-6.7(4)$ & $\mathrm{C} 32-\mathrm{C} 33-\mathrm{C} 34-\mathrm{C} 35$ & $3.5(5)$ \\
\hline $\mathrm{C} 8-\mathrm{C} 9-\mathrm{C} 14-\mathrm{C} 13$ & $-2.0(5)$ & $\mathrm{C} 32-\mathrm{C} 33-\mathrm{C} 36-\mathrm{O} 5$ & $33.9(5)$ \\
\hline $\mathrm{C} 8-\mathrm{C} 11-\mathrm{C} 12-\mathrm{C} 13$ & $-1.6(6)$ & $\mathrm{C} 33-\mathrm{C} 34-\mathrm{C} 35-\mathrm{C} 31$ & $0.0(6)$ \\
\hline $\mathrm{C} 9-\mathrm{C} 8-\mathrm{C} 11-\mathrm{C} 12$ & $-4.0(5)$ & $\mathrm{C} 34-\mathrm{C} 33-\mathrm{C} 36-\mathrm{O} 5$ & $-151.0(3)$ \\
\hline $\mathrm{C} 10-\mathrm{C} 6-\mathrm{C} 7-\mathrm{C} 2$ & $175.7(3)$ & $\mathrm{C} 36-\mathrm{O} 5-\mathrm{C} 37-\mathrm{C} 38$ & $167.2(3)$ \\
\hline
\end{tabular}




$\begin{array}{llll}\mathrm{C} 10-\mathrm{C} 9-\mathrm{C} 14-\mathrm{C} 13 & -178.2(4) & \mathrm{C} 36-\mathrm{O} 5-\mathrm{C} 37-\mathrm{C} 42 & -13.0(5) \\ \mathrm{C} 11-\mathrm{C} 8-\mathrm{C} 9-\mathrm{C} 10 & -177.2(3) & \mathrm{C} 36-\mathrm{C} 33-\mathrm{C} 34-\mathrm{C} 35 & -171.7(3) \\ \mathrm{C} 11-\mathrm{C} 8-\mathrm{C} 9-\mathrm{C} 14 & 5.9(6) & \mathrm{C} 37-\mathrm{O} 5-\mathrm{C} 36-\mathrm{C} 33 & 78.4(4) \\ \mathrm{C} 11-\mathrm{C} 12-\mathrm{C} 13-\mathrm{C} 14 & 5.5(6) & \mathrm{C} 37-\mathrm{C} 38-\mathrm{C} 39-\mathrm{C} 40 & 0.1(6) \\ \mathrm{C} 11-\mathrm{C} 12-\mathrm{C} 13-\mathrm{C} 15 & -170.5(4) & \mathrm{C} 38-\mathrm{C} 37-\mathrm{C} 42-\mathrm{C} 41 & 1.3(5) \\ \mathrm{C} 12-\mathrm{C} 13-\mathrm{C} 14-\mathrm{C} 9 & -3.6(5) & \mathrm{C} 38-\mathrm{C} 39-\mathrm{C} 40-\mathrm{C} 41 & 0.0(6) \\ \mathrm{C} 12-\mathrm{C} 13-\mathrm{C} 15-\mathrm{O} 2 & 168.8(3) & \mathrm{C} 39-\mathrm{C} 40-\mathrm{C} 41-\mathrm{O} 6 & -179.6(3) \\ \mathrm{C} 14-\mathrm{C} 9-\mathrm{C} 10-\mathrm{O} 1 & -10.7(7) & \mathrm{C} 39-\mathrm{C} 40-\mathrm{C} 41-\mathrm{C} 42 & 0.5(5) \\ \mathrm{C} 14-\mathrm{C} 9-\mathrm{C} 10-\mathrm{C} 6 & 169.9(4) & \mathrm{C} 40-\mathrm{C} 41-\mathrm{C} 42-\mathrm{C} 37 & -1.2(5) \\ \mathrm{C} 14-\mathrm{C} 13-\mathrm{C} 15-\mathrm{O} 2 & -7.0(5) & \mathrm{C} 41-\mathrm{O} 6-\mathrm{C} 1-\mathrm{C} 2 & -90.6(4) \\ \mathrm{C} 15-\mathrm{O} 2-\mathrm{C} 16-\mathrm{C} 17 & 158.0(3) & \mathrm{C} 42-\mathrm{C} 37-\mathrm{C} 38-\mathrm{C} 39 & -0.7(5) \\ \mathrm{C} 15-\mathrm{O} 2-\mathrm{C} 16-\mathrm{C} 21 & -23.6(5) & \mathrm{C} 11-\mathrm{C} 43-\mathrm{C} 44-\mathrm{C} 45 & -177.9(3) \\ \mathrm{C} 15-\mathrm{C} 13-\mathrm{C} 14-\mathrm{C} 9 & 172.1(3) & \mathrm{C} 11-\mathrm{C} 43-\mathrm{C} 48-\mathrm{C} 47 & 177.0(4) \\ \mathrm{C} 16-\mathrm{O} 2-\mathrm{C} 15-\mathrm{C} 13 & 91.6(4) & \mathrm{C} 43-\mathrm{C} 44-\mathrm{C} 45-\mathrm{C} 46 & 0.5(6) \\ \mathrm{C} 16-\mathrm{C} 17-\mathrm{C} 18-\mathrm{C} 19 & -0.7(6) & \mathrm{C} 44-\mathrm{C} 43-\mathrm{C} 48-\mathrm{C} 47 & -2.2(7) \\ \mathrm{C} 17-\mathrm{C} 16-\mathrm{C} 21-\mathrm{C} 20 & -0.2(6) & \mathrm{C} 44-\mathrm{C} 45-\mathrm{C} 46-\mathrm{C} 47 & -1.4(7) \\ \mathrm{C} 17-\mathrm{C} 18-\mathrm{C} 19-\mathrm{C} 20 & 0.7(6) & \mathrm{C} 45-\mathrm{C} 46-\mathrm{C} 47-\mathrm{C} 48 & 0.5(7) \\ \mathrm{C} 18-\mathrm{C} 19-\mathrm{C} 20-\mathrm{O} 3 & -179.7(3) & \mathrm{C} 46-\mathrm{C} 47-\mathrm{C} 48-\mathrm{C} 43 & 1.3(7) \\ \mathrm{C} 18-\mathrm{C} 19-\mathrm{C} 20-\mathrm{C} 21 & -0.3(6) & \mathrm{C} 48-\mathrm{C} 43-\mathrm{C} 44-\mathrm{C} 45 & 1.3(7)\end{array}$

Hydrogen-bond geometry $\left(\AA,{ }^{\circ}\right)$

$C g 1, C g 2$ and $C g 15$ are the centroids of the $\mathrm{C} 5 / \mathrm{C} 6 / \mathrm{C} 10 / \mathrm{C} 9 / \mathrm{C} 8, \mathrm{C} 25 / \mathrm{C} 29 / \mathrm{C} 30 / \mathrm{C} 31 / \mathrm{C} 26$ and $\mathrm{C} 43-\mathrm{C} 48$ rings, respectively.

\begin{tabular}{lllll}
\hline$D-\mathrm{H}^{\prime} \cdots A$ & $D-\mathrm{H}$ & $\mathrm{H} \cdots A$ & $D \cdots A$ & $D-\mathrm{H} \cdots A$ \\
\hline $\mathrm{C} 18-\mathrm{H} 18 \cdots \mathrm{C} 11^{\mathrm{i}}$ & 0.95 & 2.83 & $3.547(4)$ & 133 \\
$\mathrm{C} 35-\mathrm{H} 35 \cdots \mathrm{O} 1^{\mathrm{ii}}$ & 0.95 & 2.58 & $3.491(5)$ & 161 \\
$\mathrm{C} 46-\mathrm{H} 46 \cdots \mathrm{O} 6^{\mathrm{iii}}$ & 0.95 & 2.55 & $3.418(5)$ & 152 \\
$\mathrm{C} 1-\mathrm{H} 1 A \cdots C g 2^{\text {iv }}$ & 0.99 & 2.95 & $3.610(4)$ & 125 \\
$\mathrm{C} 22-\mathrm{H} 22 A \cdots C g 1^{\mathrm{v}}$ & 0.99 & 2.73 & $3.711(4)$ & 170 \\
$\mathrm{C} 36-\mathrm{H} 36 B \cdots C g 15^{\text {vi }}$ & 0.99 & 2.84 & $3.713(4)$ & 148
\end{tabular}

Symmetry codes: (i) $x+1, y+1, z$; (ii) $x-1, y, z$; (iii) $x+1, y+1, z+1$; (iv) $x, y-1, z$; (v) $x, y+1, z$; (vi) $x-1, y, z-1$. 